\title{
BACKWARD STOCHASTIC DIFFERENTIAL EQUATIONS WITH TWO DISTINCT REFLECTING BARRIERS AND QUADRATIC GROWTH GENERATOR
}

\author{
S. HAMADÈNE AND I. HDHIRI
}

Received 29 June 2004; Revised 25 January 2005; Accepted 25 January 2005

We show the existence of a solution for the double-barrier reflected BSDE when the barriers are completely separate and the generator is continuous with quadratic growth. As an application, we solve the risk-sensitive mixed zero-sum stochastic differential game. In addition we deal with recallable options under Knightian uncertainty.

Copyright (c) 2006 S. Hamadène and I. Hdhiri. This is an open access article distributed under the Creative Commons Attribution License, which permits unrestricted use, distribution, and reproduction in any medium, provided the original work is properly cited.

\section{Introduction}

A solution for a BSDE whose generator is $f(t, \omega, y, z)$ and terminal value $\xi$ is a pair of adapted processes $\left(Y_{t}, Z_{t}\right)_{t \leq T}$ which satisfies

$$
Y_{t}=\xi+\int_{t}^{T} f\left(s, \omega, Y_{s}, Z_{s}\right) d s-\int_{t}^{T} Z_{s} d B_{s}, \quad t \leq T .
$$

The adaptation is related to the natural filtration of the Brownian motion $\left(B_{t}\right)_{t \leq T}$.

In 1990, Pardoux and Peng introduced the notion of nonlinear backward stochastic differential equation (BSDE), namely (1.1), and gave existence and uniqueness result in their founder paper [26]. Since then the interest in BSDEs has kept growing steadily and there have been several works on that subject. The main reason is that BSDEs are encountered in many fields of mathematics such as finance $[6,7,31]$, stochastic games and optimal control $[4,10-12,14,15]$, partial differential equations and homogeneization [25, 27-29].

Further, other settings of BSDEs have been introduced. In [5], El-Karoui et al. consider one-barrier reflected BSDEs, that is, the situation where the process $Y$ of $(1.1)$ is forced to stay above a given barrier $\left(L_{t}\right)_{t \leq T}$. In (1.1), they add a nondecreasing continuous process $\left(K_{t}\right)_{t \leq T}$ which allows us to have $Y \geq L$. Their motivations are linked, on the one hand, to the pricing of American options and, on the other hand, to viscosity solutions 
of PDEs with an obstacle. This work has been generalized by Cvitanić and Karatzas [3] who consider the problem where the process $Y$ is now forced to stay between two comparable given barriers $L$ and $U(L \leq U)$ (see (1.2) below). In (1.1), they add a difference of nondecreasing processes $K^{+}-K^{-}$which maintain $Y$ between $L$ and $U$. Mainly, in [3], the authors show the existence and uniqueness of a solution when $f$ is Lipschitz and the barriers are either regular (they are semimartingales) or satisfy Mokobodski's condition which, roughly speaking, means that between $L$ and $U$ there exists a difference of nonnegative supermartingales.

Another direction of research which has attracted many works is the weakness of conditions under which the standard equation (1.1) has a solution. Among others, we can quote $[8,19,22,23]$. However in all of those papers, just the one-dimensional case is considered since the results are heavily based on comparison theorem which works only in that case. In [19], Kobylanski shows the existence of a solution for (1.1) when $f$ is continuous with quadratic growth with respect to $z$, that is, $|f(t, \omega, y, z)| \leq C\left(1+|y|+|z|^{2}\right)$.

So in this paper we are dealing with BSDEs with two reflecting barriers $L \leq U$ and a quadratic growth generator $f$. A solution for that equation is a quadruple of adapted processes $\left(Y, Z, K^{+}, K^{-}\right):=\left(Y_{t}, Z_{t}, K_{t}^{+}, K_{t}^{-}\right)_{t \leq T}$ with values in $\mathbb{R}^{1+d+1+1}$ which mainly satisfies the following:

$$
\begin{gathered}
K^{+} \text {and } K^{-} \text {are continuous nondecreasing processes, } \\
-d Y_{t}=f\left(t, Y_{t}, Z_{t}\right) d t+d K_{t}^{+}-d K_{t}^{-}-Z_{t} d B_{t} \quad \forall t \leq T, Y_{T}=\xi, \\
L_{t} \leq Y_{t} \leq U_{t} \quad \forall t \leq T, \quad\left(Y_{t}-L_{t}\right) d K_{t}^{+}=\left(U_{t}-Y_{t}\right) d K_{t}^{-}=0 \quad \forall t \leq T .
\end{gathered}
$$

Under Mokobodski's condition on the barriers, this equation has been considered by Bahlali et al. in [1]. They show the existence of a solution. However the trouble is that Mokobodski's condition is not easy to check in practice. Therefore the main goal of this paper is to give conditions on the barriers $L$ and $U$, as general as possible (we could say minimal) which are easy to verify in practice under which the BSDE (1.2) has a solution when $f$ is of quadratic growth in $z$. We mainly show that it is enough to have the barriers completely separated, that is, for any $t \leq T, L_{t}<U_{t}$. In addition the solution we have constructed is maximal.

BSDEs with quadratic growth coefficients and just one reflecting barrier have been considered in [20].

This paper is organized as follows. In Section 2, we recall some results related to BSDEs whose generators are continuous with linear growth. We give comparison results which play a crucial role in the proof of the main theorem. Section 3 is devoted to the proof of the main result. As in the nonreflected case, we use an exponential transform in order to obtain a BDSE whose generator satisfies a structure condition. Afterwards we show that this BSDE has a solution. Finally we obtain a solution for the initial equation by using a logarithmic transform. In addition it is maximal. The main difficulty stems from the lack of integrability of the processes. It is overcome by using a Cantor diagonal procedure. In Section 4, we use the results of Section 3 in order to solve the risk-sensitive zero-sum 
mixed stochastic differential game. Briefly the game problem which we consider is as follows.

Assume we have a system $\mathbf{S}$ on which two agents $c_{1}$ and $c_{2}$ intervene. The interventions of the agents have two forms, control and stopping, that is, they control $\mathbf{S}$ up to the time when one of them decides to stop controlling. Their actions are not free and their advantages are antagonistic, that is, there exits a payoff $J$, which depends on the implemented strategies, which is a reward for $c_{2}$ and a cost for $c_{1}$. On the other hand, the expression of $J$ integrates sensitiveness with respect to risk of the agents, either they are risk-seeking or risk-averse, by the mean of an exponential utility function. Our objective is to show existence of a fair strategy, to be precise a saddle-point, for the game and to characterize it.

We show that with this zero-sum game problem, a BSDE with two reflecting barriers of the type studied in Section 3 is associated. This BSDE gives the value function of the game and allows us to construct a saddle-point. Finally, we deal with an example for which we give a numerical result.

In Section 5, we deal with the yield of an American game or recallable option under Knightian uncertainty and exponential utility function. We characterize the lower (resp., upper) yield by means of BSDEs with quadratic growth coefficient and we study an example for which we give also a numerical result.

\section{Preliminaries}

Throughout this paper $(\Omega, \mathscr{F}, P)$ is a fixed probability space in which is defined a standard $d$-dimensional Brownian motion $B=\left(B_{t}\right)_{t \leq T}$ whose natural filtration is $\left(F_{t}^{0}:=\sigma\left\{B_{s}, s \leq\right.\right.$ t\} $)_{t \leq T} ;\left(F_{t}\right)_{t \leq T}$ is the completed filtration of $\left(F_{t}^{0}\right)_{t \leq T}$ with the $P$-null sets of $\mathscr{F}_{F}$, hence $\left(F_{t}\right)_{t \leq T}$ satisfies the usual conditions, that is, it is right continuous and complete. On the other hand, let

(i) $\mathscr{P}$ be the $\sigma$-algebra on $[0, T] \times \Omega$ of $F_{t}$-progressively measurable sets;

(ii) $\mathscr{T}$ the set of stopping times;

(iii) $\mathscr{L}^{2, k}$ the set of $\mathscr{P}$-measurable and $\mathbb{R}^{k}$-valued processes $v=\left(v_{t}\right)_{t \leq T}$ such that $\int_{0}^{T}\left|v_{s}\right|^{2} d s<\infty, P$ - a.s.;

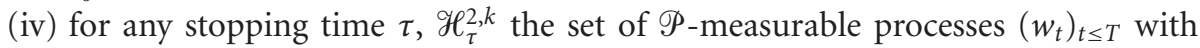
values in $\mathbb{R}^{k}$ and such that $E\left[\int_{0}^{\tau}\left|w_{s}\right|^{2} d s\right]<\infty ; \mathscr{H}_{T}^{2, k}$ will be simply denoted $\mathscr{H}^{2, k}$;

(v) $\mathscr{S}^{2}$ the set of $\mathscr{P}$-measurable and continuous processes $Y=\left(Y_{t}\right)_{t \leq T}$ such that $E\left[\sup _{t \leq T}\left|Y_{t}\right|^{2}\right]$ is finite;

(vi) $\mathcal{M}$ the set of continuous $\mathscr{P}$-measurable nondecreasing processes $\left(K_{t}\right)_{t \leq T}$ such that $K_{0}=0$ and $K_{T}<\infty, P$ - a.s.

Now we are given four objects:

(i) a function $f:[0, T] \times \Omega \times \mathbb{R}^{1+d} \rightarrow \mathbb{R}$ such that for any $(y, z) \in \mathbb{R}^{1+d}$, the process $(f(t, y, z))_{t \leq T}$ is $\mathscr{P}$-measurable and $(f(t, \omega, 0,0))_{t \leq T}$ belongs to $\mathscr{H}^{2,1}$;

(ii) a terminal value $\xi$ which is a random variable $F_{T}$-measurable and $E\left[|\xi|^{2}\right]<\infty$;

(iii) two processes $U:=\left(U_{t}\right)_{t \leq T}$ and $L:=\left(L_{t}\right)_{t \leq T}$ which belong to $\mathscr{S}^{2}$ and satisfy $L_{t}<$ $U_{t}$, for all $t \leq T$, and $L_{T} \leq \xi \leq U_{T}$.

A solution for the two reflecting barriers BSDE associated with $(f, \xi, L, U)$ is a quadruple of $\mathscr{P}$-measurable processes $\left(Y, Z, K^{+}, K^{-}\right):=\left(Y_{t}, Z_{t}, K_{t}^{+}, K_{t}^{-}\right)_{t \leq T}$ with values in $\mathbb{R}^{1+d+1+1}$ 
such that

$$
\begin{gathered}
Y \in \mathscr{Y}^{2}, \quad Z \in \mathscr{L}^{2, d}, \quad K^{+}, K^{-} \in \mathcal{M} \\
Y_{t}=\xi+\int_{t}^{T} f\left(s, Y_{s}, Z_{s}\right) d s+\left(K_{T}^{+}-K_{t}^{-}\right)-\left(K_{T}^{-}-K_{t}^{-}\right)-\int_{t}^{T} Z_{s} d B_{s} \quad \forall t \leq T, \\
L_{t} \leq Y_{t} \leq U_{t} \quad \forall t \leq T, \quad \int_{0}^{T}\left(Y_{s}-L_{s}\right) d K_{s}^{+}=\int_{0}^{T}\left(U_{s}-Y_{s}\right) d K_{s}^{-}=0 .
\end{gathered}
$$

Let us point out that in our setting, contrary to those of some other works on the same subject (see, e.g., $[1,3,13]$ ), we require neither that $Z \in \mathscr{H}^{2, d}$ nor $K^{ \pm} \in \mathscr{Y}^{2}$. The main reason is that in many applications where those equations rise up, such as stochastic games or mathematical finance, we do not need such properties for $Z$ and $K^{ \pm}$. Therefore we have adopted our setting which is less narrow than the ones of the works quoted above.

Now let us recall some known results for BSDEs whose generators $f$ are continuous with respect to $(y, z)$ and which grow sublinearly, that is,

(A1) $f$ is continuous with respect to $(y, z)$ and there exists a constant $C$ such that

$$
\text { P-a.s., } \quad|f(t, w, y, z)| \leq C(1+|y|+|z|) \quad \text { for any }(t, y, z) \in[0, T] \times \mathbb{R}^{1+d} \text {. }
$$

We have the following result.

Theorem 2.1 [9]. Assume that (A1) holds. Then the double-barrier reflected BSDE associated with $(f, \xi, L, U)$ has a maximal solution, that is, there exists a quadruple $\left(Y_{t}, Z_{t}, K_{t}^{+}\right.$, $\left.K_{t}^{-}\right)_{t \leq T}$ which satisfies (2.1). In addition if $\left(Y_{t}^{\prime}, Z_{t}^{\prime}, K_{t}^{\prime+}, K_{t}^{\prime-}\right)$ is another solution of (2.1), then $P$-a.s. holds for all $t \leq T, Y_{t} \geq Y_{t}^{\prime}$.

Let $f^{\prime}$ be another function which satisfies (A1) and such that $\left(f^{\prime}(t, y, z)\right)_{t \leq T}$ is $\mathscr{P}_{-}$ measurable for any $(y, z) \in \mathbb{R}^{1+d}$. If we can compare $f$ and $f^{\prime}$, then we can compare the maximal solutions of the double-barrier reflected BSDEs associated with $(f, \xi, L, U)$ and $\left(f^{\prime}, \xi, L, U\right)$, respectively. Namely we have the following result.

Proposition $2.2[1,9]$. Assume that P-a.s. $f(t, w, y, z) \leq f^{\prime}(t, w, y, z)$, for any $t, y$, and $z$. Let $\left(Y_{t}, Z_{t}, K_{t}^{+}, K_{t}^{-}\right)_{t \leq T}$ (resp., $\left.\left(Y_{t}^{\prime}, Z_{t}^{\prime}, K_{t}^{\prime+}, K_{t}^{\prime-}\right)_{t \leq T}\right)$ be the maximal solution of the doublereflected barrier BSDE associated with $(f, \xi, L, U)$ (resp., $\left.\left(f^{\prime}, \xi^{\prime}, L^{\prime}, U^{\prime}\right)\right)$, then $P$ - a.s., $Y \leq$ $Y^{\prime}$. In addition if $L \equiv L^{\prime}$ and $U \equiv U^{\prime}$, then also $K^{+} \geq K^{\prime+}$ and $K^{-} \leq K^{\prime-}, P-$ a.s.

In Theorem 2.1 above we already have $K_{T}^{ \pm}<\infty P$ - a.s., but we do not know whether $E\left[K_{T}^{ \pm}\right]$is finite or not. However there is some kind of local integrability for $K^{ \pm}$and $Z$. Actually we have the following.

Proposition 2.3 [9]. There exists an increasing sequence of stopping times $\left(\gamma_{k}\right)_{k \geq 0}$ such that

(i) $P$ - a.s., the sequence is stationary and converges to $T$;

(ii) the sequence depends only on $L, U$, and the constant $C$ quoted in (A1);

(iii) for all $k \geq 0$,

$$
E\left[K_{\gamma_{k}}^{+2}+K_{\gamma_{k}}^{-2}+\int_{0}^{\gamma_{k}}\left|Z_{s}^{2}\right| d s\right]<\infty .
$$




\section{Double-barrier reflected BSDE with quadratic growth generator}

From now on, we assume that $U, L$, and $\xi$ are bounded, that is, there exists a constant $C^{\prime} \geq 0$ such that

$$
P \text {-a.s. } \quad \forall t \leq T, \quad|\xi|+\sup _{t \leq T}\left\{\left|U_{t}\right|+\left|L_{t}\right|\right\} \leq C^{\prime} .
$$

On the other hand we assume that $f$ is continuous and grows subquadratically with respect to $z$, that is, it satisfies

(A2) $f$ is continuous and there exists a constant $C \geq 0$ such that

$$
\text { P-a.s., } \quad|f(t, y, z)| \leq C\left(1+|z|^{2}\right) \quad \text { for any }(t, y, z) \in[0, T] \times \mathbb{R}^{1+d} .
$$

3.1. Existence of a solution. Our aim in this section is to prove the existence of a solution for the double-barrier reflected BSDE associated with $(f, \xi, L, U)$ under assumption (A2), that is, there exists a quadruple $\left(Y_{t}, Z_{t}, K_{t}^{+}, K_{t}^{-}\right)_{t \leq T}$ which satisfies

$$
\begin{gathered}
Y \in \mathscr{S}^{2}, \quad Z \in \mathscr{L}^{2, d}, \quad K^{+}, K^{-} \in \mathcal{M}, \\
Y_{t}=\xi+\int_{t}^{T} f\left(s, Y_{s}, Z_{s}\right) d s+\left(K_{T}^{+}-K_{t}^{+}\right)-\left(K_{T}^{-}-K_{t}^{-}\right)-\int_{t}^{T} Z_{s} d B_{s} \quad \forall t \leq T, \\
L_{t} \leq Y_{t} \leq U_{t} \quad \forall t \leq T, \quad \int_{0}^{T}\left(Y_{s}-L_{s}\right) d K_{s}^{+}=\int_{0}^{T}\left(U_{s}-Y_{s}\right) d K_{s}^{-}=0 .
\end{gathered}
$$

To do so we are going first to show the existence of a solution for the reflected BSDE associated with data obtained by an exponential transform of $(f, \xi, L, U)$. More precisely let us set $m_{0}=\operatorname{essinf}_{t, w} L_{t}(w), M_{0}=\operatorname{esssup}_{t, \omega} U_{t}(\omega), m=\exp \left(2 C m_{0}\right)$, and $M=\exp \left(2 C M_{0}\right)$ where $C$ is the constant of $(\mathrm{A} 2)$. Now for $(t, y, z) \in[0, T] \times\left[m, \infty\left[\times \mathbb{R}^{d}\right.\right.$, let us set

$$
F(t, w, y, z)=2 C y\left[f\left(t, w, \frac{\ln y}{2 C}, \frac{z}{2 C y}\right)-\frac{\left|z^{2}\right|}{4 C y^{2}}\right] .
$$

Then from (A2), the function $F$ satisfies the following structure condition:

(A3) there exists a positive constant $\alpha \geq C$ such that

$$
\text { P-a.s. }-2 \alpha^{2} y-\alpha|z|^{2} \leq F(t, w, y, z) \leq 2 C^{2} y \quad \forall(t, y, z) \in[0, T] \times\left[m, \infty\left[\times \mathbb{R}^{d} .\right.\right.
$$

Now to begin with we will prove that the $\mathrm{BSDE}$ associated with $(F, \exp (2 C \xi), \exp (2 C L)$, $\exp (2 C U))$ has a solution. So let us set $\eta=\exp (2 C \xi), L^{\prime}=\exp (2 C L)$, and $U^{\prime}=\exp (2 C U)$. Then we have the following result.

Theorem 3.1. The double-barrier reflected BSDE associated with $\left(F, \eta, L^{\prime}, U^{\prime}\right)$

$$
\begin{gathered}
Y \in \mathscr{S}^{2}, \quad Z \in \mathscr{L}^{2, d}, \quad K^{+}, K^{-} \in \mathcal{M}, \\
Y_{t}=\eta+\int_{t}^{T} F\left(s, Y_{s}, Z_{s}\right) d s+\left(K_{T}^{+}-K_{t}^{+}\right)-\left(K_{T}^{-}-K_{t}^{-}\right)-\int_{t}^{T} Z_{s} d B_{s} \quad \forall t \leq T, \\
L_{t}^{\prime} \leq Y_{t} \leq U_{t}^{\prime} \quad \forall t \leq T, \quad \int_{0}^{T}\left(Y_{s}-L_{s}^{\prime}\right) d K_{s}^{+}=\int_{0}^{T}\left(U_{s}^{\prime}-Y_{s}\right) d K_{s}^{-}=0
\end{gathered}
$$

has a maximal solution $\left(Y_{t}, Z_{t}, K_{t}^{+}, K_{t}^{-}\right)_{t \leq T}$. 
Proof. Let $\rho$ be the function from $\mathbb{R}$ into $\mathbb{R}$ such that for any $y \in \mathbb{R}, \rho(y)=m 1_{[y<m]}+$ $y 1_{[m \leq y \leq M]}+M 1_{[y>M]}$, where $m$ and $M$ are the constants previously defined. On the other hand, let $k_{p}$ be a smooth function from $\mathbb{R}^{d}$ into $\mathbb{R}^{+}$such that

$$
0 \leq k_{p} \leq 1, \quad k_{p}(z)=1 \quad \text { if }|z| \leq p, \quad k_{p}(z)=0 \quad \text { if }|z| \geq p+1 .
$$

Now let $\widetilde{F}$ be the function defined by $\widetilde{F}(t, \omega, y, z)=F(t, \omega, \rho(y), z)$ for any $(t, y, z) \in[0$, $T] \times \mathbb{R}^{1+d}$. We are going to show that the following reflected BSDE:

$$
\begin{gathered}
Y \in \mathscr{Y}^{2}, \quad Z \in \mathscr{L}^{2, d}, \quad K^{+}, K^{-} \in \mathcal{M}, \\
Y_{t}=\eta+\int_{t}^{T} \tilde{F}\left(s, Y_{s}, Z_{s}\right) d s+\left(K_{T}^{+}-K_{t}^{+}\right)-\left(K_{T}^{-}-K_{t}^{-}\right)-\int_{t}^{T} Z_{s} d B_{s} \quad \forall t \leq T, \\
L_{t}^{\prime} \leq Y_{t} \leq U_{t}^{\prime} \quad \forall t \leq T, \quad \int_{0}^{T}\left(Y_{s}-L_{s}^{\prime}\right) d K_{s}^{+}=\int_{0}^{T}\left(U_{s}^{\prime}-Y_{s}\right) d K_{s}^{-}=0
\end{gathered}
$$

has a maximal solution $\left(Y_{t}, Z_{t}, K_{t}^{+}, K_{t}^{-}\right)_{t \leq T}$. Therefore it holds true that $m \leq Y \leq M$ and then $\left(Y_{t}, Z_{t}, K_{t}^{+}, K_{t}^{-}\right)_{t \geq T}$ is obviously a maximal solution for (3.6).

So for $p \geq 0$ let us set $\widetilde{F}^{p}(t, \omega, y, z)=2 C^{2} \rho(y)\left(1-k_{p}(z)\right)+k_{p}(z) \tilde{F}(t, \omega, y, z)$ for any $t, y$, and $z$. Therefore it is easily seen that $\tilde{F}^{p}$ is continuous and bounded. In addition through (3.5) and the definition of $k_{p}$, the sequence $\left(\tilde{F}^{p}\right)_{p \geq 0}$ is decreasing and $\lim _{p \rightarrow \infty} \widetilde{F}^{p}=\tilde{F}$.

Now for $p \geq 0$ let $\left(Y_{t}^{p}, Z_{t}^{p}, K_{t}^{p_{+}}, K_{t}^{p_{-}}\right)_{t \leq T}$ be the maximal solution of the double-barrier reflected BSDE associated with $\left(\tilde{F}^{p}, \eta, L^{\prime}, U^{\prime}\right)$ which exists according to Theorem 2.1. Then we have

$$
\begin{gathered}
Y^{p} \in \mathscr{Y}^{2}, \quad Z^{p} \in \mathscr{L}^{2, d}, \quad K^{p+}, K^{p-} \in \mathcal{M}, \\
Y_{t}^{p}=\eta+\int_{t}^{T} \tilde{F}^{p}\left(s, Y_{s}^{p}, Z_{s}^{p}\right) d s+\left(K_{T}^{p+}-K_{t}^{p+}\right)-\left(K_{T}^{p-}-K_{t}^{p-}\right)-\int_{t}^{T} Z_{s}^{p} d B_{s} \quad \forall t \leq T, \\
L_{t}^{\prime} \leq Y_{t}^{p} \leq U_{t}^{\prime} \quad \forall t \leq T, \quad \int_{0}^{T}\left(Y_{s}^{p}-L_{s}^{\prime}\right) d K_{s}^{p+}=\int_{0}^{T}\left(U_{s}^{\prime}-Y_{s}^{p}\right) d K_{s}^{p-}=0 .
\end{gathered}
$$

As $\widetilde{F}^{p} \geq \widetilde{F}^{p+1}$, then according to the comparison result of Proposition 2.2 we have $Y^{p} \geq$ $Y^{p+1}, K^{p+} \leq K^{p+1+}$, and $K^{p-} \geq K^{p+1-}$. On the other hand since $L_{t}^{\prime} \leq Y_{t}^{p} \leq U_{t}^{\prime}$ and through the boundedness of $L^{\prime}, U^{\prime}$, there exits an upper semicontinuous process $\left(Y_{t}\right)_{t \leq T}$ such that $P$-a.s., for all $t \leq T, Y_{t}=\lim _{p \rightarrow \infty} Y_{t}^{p}$. In addition the sequence $\left(Y^{p}\right)_{p \geq 0}$ converges in $\mathcal{H}^{2,1}$ to $\left(Y_{t}\right)_{t \leq T}$.

Now let $\left(Y_{t}^{0}, Z_{t}^{0}, K_{t}^{0+}, K_{t}^{0-}\right)_{t \leq T}$ be the maximal solution of the reflected BSDE associated with $\left(\widetilde{F}^{0}, \eta, L^{\prime}, U^{\prime}\right)$, then we have $K^{p^{-}} \leq K^{0^{-}} P$-a.s. In addition, since $\widetilde{F}^{0}$ is continuous and bounded, then there exists a nondecreasing stationary sequence of stopping times $\left(\gamma_{k}\right)_{k \geq 0}$, depending only on $L^{\prime}, U^{\prime}$, and the constant $C$ of boundedness of $\widetilde{F}^{0}$ and converging to $T$, such that

$$
E\left[\left(K_{\gamma_{k}}^{0+}\right)^{2}+\left(K_{\gamma_{k}}^{0-}\right)^{2}+\int_{0}^{\gamma_{k}}\left|Z_{s}^{0}\right|^{2} d s\right]<\infty \quad \forall k \geq 0
$$

The rest of the proof will be divided into five steps. 
Step 1. For any $p \geq 0$ and $k \geq 0$, there exists a constant $C_{k}$ which depends only on $k$ such that

$$
E\left[K_{\gamma_{k}}^{p^{+}}+K_{\gamma_{k}}^{p^{-}}+\int_{0}^{\gamma_{k}}\left|Z_{s}^{p}\right|^{2} d s\right]<C_{k}
$$

Actually the sequence $\left(K^{p^{-}}\right)_{p \geq 0}$ is decreasing then $K^{p^{-}} \leq K^{0-}$ and $E\left[K_{\gamma_{k}}^{p-}\right] \leq E\left[K_{\gamma_{k}}^{0-}\right]$. Now let us prove that there exists a constant $K_{k}$ which may depend on $k$ such that

$$
E\left[\int_{0}^{\gamma_{k}}\left|Z_{s}^{p}\right|^{2} d s\right] \leq K_{k} \quad \forall p \geq 0
$$

For any $t \leq T$, we have

$$
Y_{t \wedge \gamma_{k}}^{p}=Y_{0}^{p}-\int_{0}^{t \wedge \gamma_{k}} \tilde{F}^{p}\left(s, Y_{s}^{p}, Z_{s}^{p}\right) d s-K_{t \wedge \gamma_{k}}^{p+}+K_{t \wedge \gamma_{k}}^{p-}+\int_{0}^{t \wedge \gamma_{k}} Z_{s}^{p} d B_{s}
$$

Let $\psi(x)=\exp (-3 \alpha x)$ where $\alpha$ is the constant of (3.5). Using Itô's formula with $\psi\left(Y_{t \wedge \gamma_{k}}^{p}\right)$ and taking $t=T$ we obtain

$$
\begin{aligned}
\psi\left(Y_{\gamma_{k}}^{p}\right)= & \psi\left(Y_{0}^{p}\right)-\int_{0}^{\gamma_{k}} \psi^{\prime}\left(Y_{s}^{p}\right) \tilde{F}^{p}\left(s, Y_{s}^{p}, Z_{s}^{p}\right) d s-\int_{0}^{\gamma_{k}} \psi^{\prime}\left(Y_{s}^{p}\right) d K_{s}^{p^{+}} \\
& +\int_{0}^{\gamma_{k}} \psi^{\prime}\left(Y_{s}^{p}\right) d K_{s}^{p-}+\int_{0}^{\gamma_{k}} \psi^{\prime}\left(Y_{s}^{p}\right) Z_{s}^{p} d B_{s}+\frac{1}{2} \int_{0}^{\gamma_{k}} \psi^{\prime \prime}\left(Y_{s}^{p}\right)\left|Z_{s}^{p}\right|^{2} d s .
\end{aligned}
$$

But $\psi^{\prime}<0$ and then $\int_{0}^{\gamma_{k}} \psi^{\prime}\left(Y_{s}^{p}\right) d K_{s}^{p^{+}} \leq 0$. In addition, we have $-E\left[\int_{0}^{\gamma_{k}} \psi^{\prime}\left(Y_{s}^{p}\right) d K_{s}{ }^{p^{-}}\right] \leq$ $M_{1} E\left[K_{\gamma_{k}}^{p^{-}}\right]$, where $M_{1}=\sup _{m \leq x \leq M}\left|\psi^{\prime}(x)\right|$. Now by taking expectations in both handsides of (3.14) we obtain (at least after using a localization argument and Fatou's lemma)

$$
\frac{1}{2} E\left[\int_{0}^{\gamma_{k}} \psi^{\prime \prime}\left(Y_{s}^{p}\right)\left|Z_{s}^{p}\right|^{2} d s\right] \leq E\left[\psi\left(Y_{\gamma_{k}}^{p}\right)\right]+E\left[\int_{0}^{\gamma_{k}} \psi^{\prime}\left(Y_{s}^{p}\right) \tilde{F}^{p}\left(s, Y_{s}^{p}, Z_{s}^{p}\right) d s\right]+M_{1} E\left[K_{\gamma_{k}}^{p-}\right]
$$

since $\psi \geq 0$. Now let $A=2 \alpha^{2} M$. Using the facts that $\tilde{F}$ satisfies (A3), $\widetilde{F} \leq \widetilde{F}^{p}$ for all $p \geq 0$, and that $\psi^{\prime} \leq 0$ yields

$$
\frac{1}{2} E\left[\int_{0}^{\gamma_{k}} \psi^{\prime \prime}\left(Y_{s}^{p}\right)\left|Z_{s}^{p}\right|^{2} d s\right] \leq E\left[\psi\left(Y_{\gamma_{k}}^{p}\right)\right]-E\left[\int_{0}^{\gamma_{k}} \psi^{\prime}\left(Y_{s}^{p}\right)\left\{A+\alpha\left|Z_{s}^{p}\right|^{2}\right\} d s\right]+M_{1} E\left[K_{\gamma_{k}}^{p-}\right] .
$$


8 BSDE with distinct barriers and QG generator

Therefore

$$
\begin{aligned}
& E\left[\int_{0}^{\gamma_{k}}\left[\frac{1}{2} \psi^{\prime \prime}\left(Y_{s}^{p}\right)+\alpha \psi^{\prime}\left(Y_{s}^{p}\right)\right]\left|Z_{s}^{p}\right|^{2} d s\right] \\
& \quad \leq E\left[\psi\left(Y_{\gamma_{k}}^{p}\right)\right]-A E\left[\int_{0}^{\gamma_{k}} \psi^{\prime}\left(Y_{s}^{p}\right) d s\right]+M_{1} E\left[K_{\gamma_{k}}^{p-}\right] .
\end{aligned}
$$

Now since $\psi$ is decreasing, $m \leq Y^{p} \leq M,(1 / 2) \psi^{\prime \prime}+\alpha \psi^{\prime}=(3 / 2) \alpha^{2} \psi$, and $K^{p-} \leq K^{0-}$ for all $p \geq 0$, we get

$$
\frac{3}{2} \alpha^{2} \psi(M) E\left[\int_{0}^{\gamma_{k}}\left|Z_{s}^{p}\right|^{2} d s\right] \leq \psi(m)+M_{1}\left(A E\left[\gamma_{k}\right]+E\left[K_{\gamma_{k}}^{0-}\right]\right)
$$

and then

$$
\frac{3}{2} \alpha^{2} \exp (-3 \alpha M) E\left[\int_{0}^{\gamma_{k}}\left|Z_{s}^{p}\right|^{2} d s\right] \leq \exp (-3 \alpha m)+M_{1}\left(A T+E\left[K_{\gamma_{k}}^{0-}\right]\right)
$$

Henceforth there exists a constant $K_{k}$ which depends on $k$ such that

$$
\forall p \geq 0, \quad E\left[\int_{0}^{\gamma_{k}}\left|Z_{s}^{p}\right|^{2} d s\right] \leq K_{k}
$$

Thus, it remains to show that $E\left[K_{\gamma_{k}}^{p+}\right]<\infty$. But using Itô's formula with $\exp \left(Y_{t \wedge \gamma_{k}}^{p}\right)$ and taking $t=T$, we obtain that for all $k, p \geq 0$,

$$
\begin{aligned}
\exp \left(Y_{\gamma_{k}}^{p}\right)= & \exp \left(Y_{0}^{p}\right)-\int_{0}^{\gamma_{k}} \exp \left(Y_{s}^{p}\right) \tilde{F}^{p}\left(s, Y_{s}^{p}, Z_{s}^{p}\right) d s-\int_{0}^{\gamma_{k}} \exp \left(Y_{s}^{p}\right) d K_{s}^{p+} \\
& +\int_{0}^{\gamma_{k}} \exp \left(Y_{s}^{p}\right) d K_{s}^{p-}+\int_{0}^{\gamma_{k}} \exp \left(Y_{s}^{p}\right) Z_{s}^{p} d B_{s}+\frac{1}{2} \int_{0}^{\gamma_{k}} \exp \left(Y_{s}^{p}\right)\left|Z_{s}^{p}\right|^{2} d s .
\end{aligned}
$$

Now taking expectations in both hand-sides, we obtain

$$
\begin{aligned}
E\left[\exp \left(Y_{\gamma_{k}}^{p}\right)\right]+E\left[\int_{0}^{\gamma_{k}} \exp \left(Y_{s}^{p}\right) d K_{s}^{p^{+}}\right] \\
=E\left[\exp \left(Y_{0}^{p}\right)\right]-E\left[\int_{0}^{\gamma_{k}} \exp \left(Y_{s}^{p}\right) \tilde{F}^{p}\left(s, Y_{s}^{p}, Z_{s}^{p}\right) d s\right] \\
\quad+E\left[\int_{0}^{\gamma_{k}} \exp \left(Y_{s}^{p}\right) d K_{s}^{p-}\right]+\frac{1}{2} E\left[\int_{0}^{\gamma_{k}} \exp \left(Y_{s}^{p}\right)\left|Z_{s}^{p}\right|^{2} d s\right] .
\end{aligned}
$$


But since $-\widetilde{F}^{p}\left(s, Y_{s}^{p}, Z_{s}^{p}\right) \leq-\tilde{F}\left(s, Y_{s}^{p}, Z_{s}^{p}\right) \leq 2 \alpha^{2} \rho\left(Y_{s}^{p}\right)+\alpha\left|Z_{s}^{p}\right|^{2}$ for all $s \leq T, m \leq Y^{p} \leq M$, and $\gamma_{k} \leq T$, we get

$$
\exp (m) E\left[K_{\gamma_{k}}^{p^{+}}\right] \leq \exp (M)\left\{1+2 \alpha^{2} M T+E\left[K_{\gamma_{k}}^{p^{-}}\right]+(1+\alpha) E\left[\int_{0}^{\gamma_{k}}\left|Z_{s}^{p}\right|^{2}\right] d s\right\}
$$

Finally taking into account (3.12), we obtain the desired result.

The following step is the main one in the proof of this theorem.

Step 2. There exists a subsequence of $\left(Z^{p}\right)_{p \geq 0}$ and a process $Z \in \mathscr{L}^{2, d}$ such that for any $k \geq 0,\left(Z^{p} 1_{\left[0, \gamma_{k}\right]}\right)_{p \geq 0}$ converges strongly in $\mathscr{H}^{2, d}$ to $Z 1_{\left[0, \gamma_{k}\right]}$.

Indeed for any $k \geq 0$, the sequence $\left(Z^{p} 1_{\left[0, \gamma_{k}\right]}\right)_{p \geq 0}$ is bounded in $\mathscr{H}^{2, d}$ then, through the Cantor diagonal procedure, there exists a subsequence of $\left(Z^{p}\right)_{p \geq 0}$ which we still denote $\left(Z^{p}\right)_{p \geq 0}$ such that for any $k \geq 0$, the sequence $\left(Z^{p} 1_{\left[0, \gamma_{k}\right]}\right)_{p \geq 0}$ converges weakly to a process $Z^{(k)}$ which belongs to $\mathscr{H}^{2, d}$. So let us show that $Z^{(k+1)} 1_{\left[0, \gamma_{k}\right]}=Z^{(k)} 1_{\left[0, \gamma_{k}\right]}$. Actually we have

$$
Z^{p} 1_{\left[0, \gamma_{k+1}\right]} \longrightarrow Z^{(k+1)} \quad \text { as } p \longrightarrow \infty
$$

But we have also

$$
Z^{p} 1_{\left[0, \gamma_{k}\right]}=Z^{p} 1_{\left[0, \gamma_{k+1}\right]} 1_{\left[0, \gamma_{k}\right]} \longrightarrow Z^{(k+1)} 1_{\left[0, \gamma_{k}\right]} \text { as } p \longrightarrow \infty
$$

Then uniqueness of the weak limit implies that

$$
Z^{(k+1)} 1_{\left[0, \gamma_{k}\right]}=Z^{(k)} 1_{\left[0, \gamma_{k}\right]}
$$

Now for any $t \leq T$, let us set

$$
Z_{t}=Z_{t}^{(0)} 1_{\left[0, \gamma_{0}\right]}+\sum_{k \geq 1} Z_{t}^{(k)} 1_{] \gamma_{k-1}, \gamma_{k}\right]}
$$

Since the sequence $\left(\gamma_{k}\right)_{k \geq 0}$ is of stationary type and for any $k \geq 0, E\left[\int_{0}^{\gamma_{k}}\left|Z_{s}\right|^{2} d s\right]<\infty$, then $\int_{0}^{T}\left|Z_{s}\right|^{2} d s<\infty P$ - a.s. On the other hand for any $k \geq 0, Z 1_{\left[0, \gamma_{k}\right]}=Z^{(k)} 1_{\left[0, \gamma_{k}\right]}$, then $\left(Z^{p} 1_{\left[0, \gamma_{k}\right]}\right)_{p \geq 0}$ converges weakly to $Z 1_{\left[0, \gamma_{k}\right]}$ in $\mathscr{H}^{2, d}$.

Next let $\theta=8\left(2\left(C^{2}+\alpha^{2}\right) M+\alpha\right)$ and $\psi(x)=(\exp (4 \theta x)-1) / 4 \theta-x$. Let $p<q$ be two positive integers, then $Y^{p} \geq Y^{q}$. Now by applying Itô's formula and taking expectations we obtain

$$
\begin{gathered}
E\left[\psi\left(Y_{0}^{p}-Y_{0}^{q}\right)\right]+\frac{1}{2} E\left[\int_{0}^{\gamma_{k}} \psi^{\prime \prime}\left(Y_{s}^{p}-Y_{s}^{q}\right)\left|Z_{s}^{p}-Z_{s}^{q}\right|^{2} d s\right] \\
=E\left[\psi\left(Y_{\gamma_{k}}^{p}-Y_{\gamma_{k}}^{q}\right)\right]+J_{1}(p, q)+J_{2}(p, q)+J_{3}(p, q),
\end{gathered}
$$


where

$$
\begin{aligned}
& J_{1}(p, q)=E\left[\int_{0}^{\gamma_{k}} \psi^{\prime}\left(Y_{s}^{p}-Y_{s}^{q}\right)\left(\tilde{F}^{p}\left(s, Y_{s}^{p}, Z_{s}^{p}\right)-\tilde{F}^{q}\left(s, Y_{s}^{q}, Z_{s}^{q}\right)\right) d s\right], \\
& J_{2}(p, q)=E\left[\int_{0}^{\gamma_{k}} \psi^{\prime}\left(Y_{s}^{p}-Y_{s}^{q}\right) d\left(K_{s}^{p+}-K_{s}^{q+}\right)\right], \\
& J_{3}(p, q)=-E\left[\int_{0}^{\gamma_{k}} \psi^{\prime}\left(Y_{s}^{p}-Y_{s}^{q}\right) d\left(K_{s}^{p-}-K_{s}^{q-}\right)\right] .
\end{aligned}
$$

Since $K^{p+}$ (resp., $\left.K^{q+}\right)$ moves only when $Y^{p}$ (resp., $\left.Y^{q}\right)$ reaches the obstacle $L^{\prime}$, then

$$
J_{2}(p, q)=E\left[\int_{0}^{\gamma_{k}} \psi^{\prime}\left(Y_{s}^{p}-Y_{s}^{q}\right) 1_{\left\{Y_{s}^{p}=L_{s}^{\prime}\right\}} d K_{s}^{p^{+}}\right]-E\left[\int_{0}^{\gamma_{k}} \psi^{\prime}\left(Y_{s}^{p}-Y_{s}^{q}\right) 1_{\left\{Y_{s}^{q}=L_{s}^{\prime}\right\}} d K_{s}^{q+}\right] .
$$

However $Y^{q} \leq Y^{p}$ implies that $\left\{Y^{p}=L^{\prime}\right\} \subset\left\{Y^{q}=L^{\prime}\right\}$ and therefore, since $\psi^{\prime}(0)=0$,

$$
J_{2} \leq-E\left[\int_{0}^{\gamma_{k}} \psi^{\prime}\left(Y_{s}^{p}-Y_{s}^{q}\right) 1_{\left\{Y_{s}^{q}=L_{s}^{\prime}\right\}} d K_{s}^{q+}\right]
$$

But $\psi^{\prime}(x) \geq 0$ for any $x \geq 0$, then $J_{2}(p, q) \leq 0$. In the same way we can show that $J_{3}(p, q) \leq$ 0 . Finally let us deal with $J_{1}(p, q)$. First we have

$$
\begin{aligned}
\tilde{F}^{p}(s, & \left.Y_{s}^{p}, Z_{s}^{p}\right)-\tilde{F}^{q}\left(s, Y_{s}^{q}, Z_{s}^{q}\right) \\
& \leq \widetilde{F}^{p}\left(s, Y_{s}^{p}, Z_{s}^{p}\right)-\widetilde{F}\left(s, Y_{s}^{q}, Z_{s}^{q}\right) \\
& =2 C^{2} \rho\left(Y^{p}\right)\left(1-k_{p}\left(Z^{p}\right)\right)+k_{p}\left(Z^{p}\right) \tilde{F}\left(s, Y_{s}^{p}, Z_{s}^{p}\right)-\tilde{F}\left(s, Y_{s}^{q}, Z_{s}^{q}\right) \\
& \leq 2 C^{2} \rho\left(Y_{s}^{p}\right)\left(1-k_{p}\left(Z_{s}^{p}\right)\right)+k_{p}\left(Z_{s}^{p}\right) \tilde{F}\left(s, Y_{s}^{p}, Z_{s}^{p}\right)+2 \alpha^{2} \rho\left(Y_{s}^{q}\right)+\alpha\left|Z_{s}^{q}\right|^{2} \\
& \leq 2 C^{2} \rho\left(Y_{s}^{p}\right)+2 \alpha^{2} \rho\left(Y_{s}^{q}\right)+\alpha\left|Z_{s}^{q}\right|^{2} \\
& \leq \frac{\theta}{8}\left(1+\left|Z_{s}^{q}\right|^{2}\right) \leq \theta\left(1+\left|Z_{s}^{q}-Z_{s}^{p}\right|^{2}+\left|Z_{s}^{p}-Z_{s}\right|^{2}+\left|Z_{s}\right|^{2}\right) .
\end{aligned}
$$

The third inequality stems from (3.5). Now since $\psi^{\prime}\left(Y_{s}^{p}-Y_{s}^{q}\right) \geq 0$ and $\psi\left(Y_{0}^{p}-Y_{0}^{q}\right) \geq 0$, then

$$
\begin{aligned}
E\left[\int_{0}^{\gamma_{k}}\left\{\left(\frac{1}{2} \psi^{\prime \prime}-\theta \psi^{\prime}\right)\left(Y_{s}^{p}-Y_{s}^{q}\right)\right\}\left|Z_{s}^{p}-Z_{s}^{q}\right|^{2} d s\right] \leq E\left[\psi\left(Y_{\gamma_{k}}^{p}-Y_{\gamma_{k}}^{q}\right)\right] \\
+\theta E\left[\int_{0}^{\gamma_{k}} \psi^{\prime}\left(Y_{s}^{p}-Y_{s}^{q}\right) d s\right]+\theta E\left[\int_{0}^{\gamma_{k}} \psi^{\prime}\left(Y_{s}^{p}-Y_{s}^{q}\right)\left\{\left|Z_{s}^{p}-Z_{s}\right|^{2}+\left|Z_{s}\right|^{2}\right\} d s\right] .
\end{aligned}
$$

But we have

$$
\frac{1}{2} \psi^{\prime \prime}(x)-\theta \psi^{\prime}(x)=\theta \exp (4 \theta x)+\theta
$$


Then the bounded process $\left((1 / 2) \psi^{\prime \prime}-\theta \psi^{\prime}\right)^{1 / 2}\left(Y^{p}-Y^{q}\right) 1_{\left[0, \gamma_{k}\right]}$ converges, as $q \rightarrow \infty$, strongly in $\mathscr{H}^{2,1}$ to the process $\left\{\left((1 / 2) \psi^{\prime \prime}-\theta \psi^{\prime}\right)^{1 / 2}\left(Y^{p}-Y\right)\right\} 1_{\left[0, \gamma_{k}\right]}$. Hence $\left\{\left((1 / 2) \psi^{\prime \prime}-\theta \psi^{\prime}\right)^{1 / 2} \times\right.$ $\left.\left(Y^{p}-Y^{q}\right)\right\}\left(Z^{p}-Z^{q}\right) 1_{\left[0, \gamma_{k}\right]}$ converges weakly, as $q \rightarrow \infty$, to $\left\{\left((1 / 2) \psi^{\prime \prime}-\theta \psi^{\prime}\right)^{1 / 2}\left(Y^{p}\right.\right.$ $-Y)\}\left(Z^{p}-Z\right) 1_{\left[0, \gamma_{k}\right]}$. Now since for any sequence $\left(u_{n}\right)_{n \geq 0}$ of $\mathscr{H}^{2,1}$ which converges weakly to $u$ we have $\|u\|^{2} \leq \liminf _{n \rightarrow \infty}\left\|u_{n}\right\|^{2}$, then we obtain

$$
\begin{aligned}
E\left[\int_{0}^{\gamma_{k}}\right. & \left.\left\{\left(\frac{1}{2} \psi^{\prime \prime}-\theta \psi^{\prime}\right)\left(Y_{s}^{p}-Y_{s}\right)\right\}\left|Z_{s}^{p}-Z_{s}\right|^{2} d s\right] \\
\leq & \liminf _{q \rightarrow \infty} E\left[\int_{0}^{\gamma_{k}}\left\{\left(\frac{1}{2} \psi^{\prime \prime}-\theta \psi^{\prime}\right)\left(Y_{s}^{p}-Y_{s}^{q}\right)\right\}\left|Z_{s}^{p}-Z_{s}^{q}\right|^{2} d s\right] \\
\leq & E\left[\psi\left(Y_{\gamma_{k}}^{p}-Y_{\gamma_{k}}\right)\right]+\theta E\left[\int_{0}^{\gamma_{k}} \psi^{\prime}\left(Y_{s}^{p}-Y_{s}\right) d s\right] \\
& +\theta E\left[\int_{0}^{\gamma_{k}}\left\{\psi^{\prime}\left(Y_{s}^{p}-Y_{s}\right)\right\}\left\{\left|Z_{s}^{p}-Z_{s}\right|^{2}+\left|Z_{s}\right|^{2}\right\} d s\right] .
\end{aligned}
$$

Therefore

$$
\begin{aligned}
& E\left[\int_{0}^{\gamma_{k}}\left\{\left(\frac{1}{2} \psi^{\prime \prime}-2 \theta \psi^{\prime}\right)\left(Y_{s}^{p}-Y_{s}\right)\right\}\left|Z_{s}^{p}-Z_{s}\right|^{2} d s\right] \\
& \quad \leq E\left[\psi\left(Y_{\gamma_{k}}^{p}-Y_{\gamma_{k}}\right)\right]+\theta E\left[\int_{0}^{\gamma_{k}} \psi^{\prime}\left(Y_{s}^{p}-Y_{s}\right)\left(1+\left|Z_{s}\right|^{2}\right) d s\right] .
\end{aligned}
$$

Finally using the Lebesgue-dominated convergence theorem and the equality $(1 / 2) \psi^{\prime \prime}-$ $2 \theta \psi^{\prime}=2 \theta$ we deduce that

$$
\lim _{p \rightarrow \infty} E\left[\int_{0}^{\gamma_{k}}\left|Z_{s}^{p}-Z_{s}\right|^{2} d s\right]=0
$$

Step 3. The process $Y$ is continuous.

Let $k$ be fixed and let $p, q \in \mathbb{N}$ such that $p<q$. For any $t \leq T$ we have

$$
\begin{aligned}
Y_{t \wedge \gamma_{k}}^{p}-Y_{t \wedge \gamma_{k}}^{q}= & Y_{\gamma_{k}}^{p}-Y_{\gamma_{k}}^{q}+\int_{t \wedge \gamma_{k}}^{\gamma_{k}}\left[\tilde{F}^{p}\left(s, Y_{s}^{p}, Z_{s}^{p}\right)-\widetilde{F}^{q}\left(s, Y_{s}^{q}, Z_{s}^{q}\right)\right] d s+\int_{t \wedge \gamma_{k}}^{\gamma_{k}}\left(d K_{s}^{p+}-d K_{s}^{q+}\right) \\
& -\int_{t \wedge \gamma_{k}}^{\gamma_{k}}\left(d K_{s}^{p-}-d K_{s}^{q-}\right)-\int_{t \wedge \gamma_{k}}^{\gamma_{k}}\left(Z_{s}^{p}-Z_{s}^{q}\right) d B_{s} .
\end{aligned}
$$

Using Itô's formula with $\left(Y^{p}-Y^{q}\right)^{2}$ yields that for any $t \leq T$,

$$
\begin{aligned}
\left(Y_{t \wedge \gamma_{k}}^{p}-Y_{t \wedge \gamma_{k}}^{q}\right)^{2}= & \left(Y_{\gamma_{k}}^{p}-Y_{\gamma_{k}}^{q}\right)^{2}+2 \int_{t \wedge \gamma_{k}}^{\gamma_{k}}\left(Y_{s}^{p}-Y_{s}^{q}\right)\left[\tilde{F}^{p}\left(s, Y_{s}^{p}, Z_{s}^{p}\right)-\tilde{F}^{q}\left(s, Y_{s}^{q}, Z_{s}^{q}\right)\right] d s \\
& +2 \int_{t \wedge \gamma_{k}}^{\gamma_{k}}\left(Y_{s}^{p}-Y_{s}^{q}\right)\left(d K_{s}^{p+}-d K_{s}^{q+}\right)-2 \int_{t \wedge \gamma_{k}}^{\gamma_{k}}\left(Y_{s}^{p}-Y_{s}^{q}\right)\left(d K_{s}^{p-}-d K_{s}^{q-}\right) \\
& -\int_{t \wedge \gamma_{k}}^{\gamma_{k}}\left(Y_{s}^{p}-Y_{s}^{q}\right)\left(Z_{s}^{p}-Z_{s}^{q}\right) d B_{s}-\int_{t \wedge \gamma_{k}}^{\gamma_{k}}\left|Z_{s}^{p}-Z_{s}^{q}\right|^{2} d s .
\end{aligned}
$$


But

$$
\begin{aligned}
\int_{t \wedge \gamma_{k}}^{\gamma_{k}}\left(Y_{s}^{p}-Y_{s}^{q}\right)\left(d K_{s}^{p+}-d K_{s}^{q+}\right)-\int_{t \wedge \gamma_{k}}^{\gamma_{k}}\left(Y_{s}^{p}-Y_{s}^{q}\right)\left(d K_{s}^{p-}-d K_{s}^{q-}\right) \\
=-\int_{t \wedge \gamma_{k}}^{\gamma_{k}}\left(Y_{s}^{p}-L_{s}^{\prime}\right) d K_{s}^{q+}+\int_{t \wedge \gamma_{k}}^{\gamma_{k}}\left(L_{s}^{\prime}-Y_{s}^{q}\right) d K_{s}^{p+} \\
\quad-\int_{t \wedge \gamma_{k}}^{\gamma_{k}}\left(U_{s}^{\prime}-Y_{s}^{q}\right) d K_{s}^{p-}+\int_{t \wedge \gamma_{k}}^{\gamma_{k}}\left(Y_{s}^{p}-U_{s}^{\prime}\right) d K_{s}^{q-} \leq 0 .
\end{aligned}
$$

Then

$$
\begin{aligned}
\left(Y_{t \wedge \gamma_{k}}^{p}-\right. & \left.Y_{t \wedge \gamma_{k}}^{q}\right)^{2}+\int_{t \wedge \gamma_{k}}^{\gamma_{k}}\left|Z_{s}^{p}-Z_{s}^{q}\right|^{2} d s \\
\leq & \left(Y_{\gamma_{k}}^{p}-Y_{\gamma_{k}}^{q}\right)^{2}-\int_{t \wedge \gamma_{k}}^{\gamma_{k}}\left(Y_{s}^{p}-Y_{s}^{q}\right)\left(Z_{s}^{p}-Z_{s}^{q}\right) d B_{s} \\
& +2 \int_{t \wedge \gamma_{k}}^{\gamma_{k}}\left(Y_{s}^{p}-Y_{s}^{q}\right)\left|\widetilde{F}^{p}\left(s, Y_{s}^{p}, Z_{s}^{p}\right)-\widetilde{F}^{q}\left(s, Y_{s}^{q}, Z_{s}^{q}\right)\right| d s .
\end{aligned}
$$

Now by taking the supremum, then expectations in both hand-sides finally using the Burkholder-Davis-Gundy inequality, we obtain

$$
\begin{aligned}
E\left[\sup _{t \leq \gamma_{k}}\left(Y_{t}^{p}-Y_{t}^{q}\right)^{2}\right] \leq \delta\{ & E\left[\left(Y_{\gamma_{k}}^{p}-Y_{\gamma_{k}}^{q}\right)^{2}\right]+E\left[\int_{0}^{\gamma_{k}}\left|Z_{s}^{p}-Z_{s}^{q}\right|^{2} d s\right] \\
+ & \left.E\left[\int_{0}^{\gamma_{k}}\left(Y_{s}^{p}-Y_{s}^{q}\right)\left|\tilde{F}^{p}\left(s, Y_{s}^{p}, Z_{s}^{p}\right)-\widetilde{F}^{q}\left(s, Y_{s}^{q}, Z_{s}^{q}\right)\right| d s\right]\right\},
\end{aligned}
$$

where $\delta$ is an appropriate real constant. Now the sequence $\left(Z^{p} 1_{\left[0, \gamma_{k}\right]}\right)_{p \geq 0}$ converges to $Z 1_{\left[0, \gamma_{k}\right]}$ in $\mathcal{H}^{2, d}$, then there exists a subsequence of $\left(Z^{p} 1_{\left[0, \gamma_{k}\right]}\right)_{p \geq 0}$ which we still denote $\left(Z^{p} 1_{\left[0, \gamma_{k}\right]}\right)_{p \geq 0}$ such that

$$
d t \otimes d P \text { - a.s., } \quad Z^{p} 1_{\left[0, \gamma_{k}\right]} \longrightarrow{ }_{p \rightarrow \infty} Z 1_{\left[0, \gamma_{k}\right]}, \quad \widetilde{Z}_{t}^{(k)}=\sup _{p \geq 0}\left|Z^{p} 1_{\left[0, \gamma_{k}\right]}\right|^{2} \in \mathscr{H}^{2,1} .
$$

The subsequence could depend on $k$ but this fact is irrelevant since we work at fixed $k$. On the other hand, there exists a constant $\bar{\lambda}>0$ such that

$$
\left|\tilde{F}^{p}\left(t, Y_{t}^{p}, Z_{t}^{p}\right)\right| 1_{\left[0, \gamma_{k}\right]} \leq \bar{\lambda}\left(1+1_{\left[0, \gamma_{k}\right]}\left|Z_{t}^{p}\right|^{2}\right) \leq \bar{\lambda}\left(1+\sup _{p \geq 0}\left|Z_{t}^{p} 1_{\left[0, \gamma_{k}\right]}\right|^{2}\right)=\bar{\lambda}\left(1+\left|\tilde{Z}_{t}^{(k)}\right|\right) .
$$

Therefore

$$
\begin{aligned}
E\left[\sup _{t \leq \gamma_{k}}\left(Y_{t}^{p}-Y_{t}^{q}\right)^{2}\right] \leq \delta\{ & E\left[\left(Y_{\gamma_{k}}^{p}-Y_{\gamma_{k}}^{q}\right)^{2}\right]+E\left[\int_{0}^{\gamma_{k}}\left|Z_{s}^{p}-Z_{s}^{q}\right|^{2} d s\right] \\
& \left.+2 E\left[\int_{0}^{\gamma_{k}} \bar{\lambda}\left|Y_{s}^{p}-Y_{s}^{q}\right|\left(1+\left|\tilde{Z}_{s}^{(k)}\right|\right) d s\right]\right\} .
\end{aligned}
$$


Then $E\left[\sup _{t \leq \gamma_{k}}\left(Y_{t}^{p}-Y_{t}^{q}\right)^{2}\right] \rightarrow 0$ as $p, q \rightarrow \infty$. It follows that, for any $k \geq 0$ the process $\left(Y_{t \wedge \gamma_{k}}\right)_{t \leq T}$ is continuous. Now since $\left(\gamma_{k}\right)_{k \geq 0}$ is of stationary type, then the process $Y$ is continuous.

Step 4. Construction of the processes $K^{+}$and $K^{-}$.

The sequence of processes $\left(K^{p+}\right)_{p \geq 0}$ (resp., $\left.\left(K^{p-}\right)_{p \geq 0}\right)$ is nondecreasing (resp., nonincreasing). So let us set, for $t \leq T, K_{t}^{+}=\liminf _{p \rightarrow \infty} K_{t}^{p^{+}}$and $K_{t}^{-}=\lim _{p \rightarrow \infty} K_{t}^{p-}$. Since for any $p \geq 0$ and $k \geq 0, K_{t \wedge \gamma_{k}}^{p-} \leq K_{t \wedge \gamma_{k}}^{0-}$, then $E\left[K_{\gamma_{k}}^{-}\right]<\infty$. In addition the process $\left(K_{t}^{-}\right)_{t \leq T}$ is nondecreasing and upper semicontinuous.

Next for any $k \geq 0$ we have $\sup _{p \geq 0} E\left[K_{\gamma_{k}}^{p+}\right]<\infty$, then through Fatou's lemma $E\left[K_{\gamma_{k}}^{+}\right]<$ $\infty$. Therefore for any $t \leq T, K_{t \wedge \gamma_{k}}^{+}=\lim _{p \rightarrow \infty} K_{t \wedge \gamma_{k}}^{p+}$. In addition the process $\left(K_{t}^{+}\right)_{t \leq T}$ is nondecreasing and lower semicontinuous.

Step 5. The quadruple $\left(Y_{t}, Z_{t}, K_{t}^{+}, K_{t}^{-}\right)_{t \leq T}$ is a solution for the double-barrier reflected BSDE associated with $\left(\widetilde{F}, \eta, L^{\prime}, U^{\prime}\right)$.

Let $k$ be fixed and let $t \leq T$. Then

$$
Y_{t \wedge \gamma_{k}}^{p}=Y_{\gamma_{k}}^{p}+\int_{t \wedge \gamma_{k}}^{\gamma_{k}} \tilde{F}^{p}\left(s, Y_{s}^{p}, Z_{s}^{p}\right) d s+\left(K_{\gamma_{k}}^{p+}-K_{t \wedge \gamma_{k}}^{p+}\right)-\left(K_{\gamma_{k}}^{p^{-}}-K_{t \wedge \gamma_{k}}^{p-}\right)-\int_{t \wedge \gamma_{k}}^{\gamma_{k}} Z_{s}^{p} d B_{s} .
$$

Now according to Dini's theorem, $\tilde{F}^{p}(s, y, z)$ converges uniformly to $\tilde{F}(s, y, z)$ on compact subsets, then $\tilde{F}^{p}\left(s, Y_{s}^{p}, Z_{s}^{p}\right) 1_{\left[0, \gamma_{k}\right]} \rightarrow_{p \rightarrow \infty} \widetilde{F}\left(s, Y_{s}, Z_{s}\right) 1_{\left[0, \gamma_{k}\right]}, d t \otimes d P-$ a.s. But $\mid \tilde{F}^{p}\left(s, Y_{s}^{p}\right.$, $\left.Z_{s}^{p}\right) \mid 1_{\left[0, \gamma_{k}\right]} \leq \bar{\lambda}\left(1+\left|\tilde{Z}_{s}^{(k)}\right|\right) \in \mathscr{H}^{2,1}$. This implies that

$$
E\left[\int_{0}^{\gamma_{k}}\left|\tilde{F}^{p}\left(s, Y_{s}^{p}, Z_{s}^{p}\right)-\tilde{F}\left(s, Y_{s}, Z_{s}\right) d s\right|\right] \rightarrow_{p \rightarrow \infty} 0
$$

Then, by taking the limit in (3.46) as $p \rightarrow \infty$ we obtain

$$
\begin{aligned}
Y_{t \wedge \gamma_{k}}= & Y_{\gamma_{k}}+\int_{t \wedge \gamma_{k}}^{\gamma_{k}} \tilde{F}\left(s, Y_{s}, Z_{s}\right) d s+\left(K_{\gamma_{k}}^{+}-K_{t \wedge \gamma_{k}}^{+}\right)-\left(K_{\gamma_{k}}^{-}-K_{t \wedge \gamma_{k}}^{-}\right) \\
& -\int_{t \wedge \gamma_{k}}^{\gamma_{k}} Z_{s} d B_{s} .
\end{aligned}
$$

Now writing

$$
Y_{t \wedge \gamma_{k}}=Y_{0}-\int_{0}^{t \wedge \gamma_{k}} \tilde{F}\left(s, Y_{s}, Z_{s}\right) d s-K_{t \wedge \gamma_{k}}^{+}+K_{t \wedge \gamma_{k}}^{-}+\int_{0}^{t \wedge \gamma_{k}} Z_{s} d B_{s}
$$

implies that $\left(K_{t \wedge \gamma_{k}}^{+}\right)_{t \leq T}$ and $\left(K_{t \wedge \gamma_{k}}^{-}\right)_{t \leq T}$ are lower and upper semicontinuous in the same time then they are continuous. Since $\left(\gamma_{k}\right)_{k \geq 0}$ is of stationary type, then $K^{+}$and $K^{-}$are continuous on the whole interval $[0, T]$. Now $P$-a.s., for $\omega$ fixed there exists $k_{0}(\omega)$ such that for $k \geq k_{0}$ we have $\gamma_{k}(\omega)=T$, therefore for $k$ great enough we have

$$
Y_{t}=\eta+\int_{t}^{T} \tilde{F}\left(s, Y_{s}, Z_{s}\right) d s+\left(K_{T}^{+}-K_{t}^{+}\right)-\left(K_{T}^{-}-K_{t}^{-}\right)-\int_{t}^{T} Z_{s} d B_{s} .
$$


It remains to show that

$$
\int_{0}^{T}\left(Y_{s}-L_{s}^{\prime}\right) d K_{s}^{+}=\int_{0}^{T}\left(U_{s}^{\prime}-Y_{s}\right) d K_{s}^{-}=0 .
$$

We already have

$$
\int_{0}^{T}\left(Y_{s}^{p}-L_{s}^{\prime}\right) d K_{s}^{p+}=\int_{0}^{T}\left(U_{s}^{\prime}-Y_{s}^{p}\right) d K_{s}^{p-}=0
$$

But according to Dini's theorem, $P$-a.s., $K^{p+}$ (resp., $K^{p-}$ ) converges uniformly to $K^{+}$ (resp., $K^{-}$) in the interval $[0, T]$. Then $(3.51)$ is obtained from Helly's theorem (see [21, page 362]) since $\left(Y^{p}\right)_{p \geq 0}$ converges uniformly to $Y$ and taking into account (3.52). The proof of this fact can also be seen in [16].

Therefore the quadruple $\left(Y_{t}, Z_{t}, K_{t}^{+}, K_{t}^{-}\right)_{t \leq T}$ is a solution for (3.8). Now as $\rho(Y)=Y$, then $\left(Y_{t}, Z_{t}, K_{t}^{+}, K_{t}^{-}\right)_{t \leq T}$ is also a solution for (3.6).

Finally let us show that this solution is maximal. Let $\left(\bar{Y}, \bar{Z}, \bar{K}^{+}, \bar{K}^{-}\right)$be another solution for (3.6) and then also for (3.8). We have for any $p \geq 0, \widetilde{F}^{p} \geq \widetilde{F}$ then, according to Proposition 2.2 we have $Y^{p} \geq \bar{Y}$. By taking the limit as $p \rightarrow \infty$, we obtain the desired result.

We are now ready to give the main result of this section.

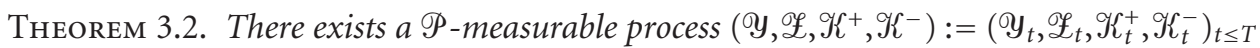
solution for the reflected BSDE associated with $(f, \xi, L, U)$, that is, which satisfies

$$
\begin{gathered}
\mathscr{y} \in \mathscr{Y}^{2}, \quad \mathscr{L} \in \mathscr{L}^{2, d}, \quad \mathscr{K}^{+}, \mathscr{K}^{-} \in \mathcal{M}, \\
\mathscr{Y}_{t}=\xi+\int_{t}^{T} f\left(s, \mathscr{Y}_{s}, \mathscr{L}_{s}\right) d s+\left(\mathscr{K}_{T}^{+}-\mathscr{Y}_{t}^{-}\right)-\left(\mathscr{K}_{T}^{-}-\mathscr{K}_{t}^{-}\right)-\int_{t}^{T} \mathscr{L}_{s} d B_{s} \quad \forall t \leq T, \\
L_{t} \leq \mathscr{Y}_{t} \leq U_{t} \quad \forall t \leq T, \quad \int_{0}^{T}\left(\mathscr{Y}_{s}-L_{s}\right) d \mathscr{K}_{s}^{+}=\int_{0}^{T}\left(U_{s}-\mathscr{Y}_{s}\right) d \mathcal{K}_{s}^{-}=0 .
\end{gathered}
$$

Moreover it is maximal.

Proof. Let $\left(Y_{t}, Z_{t}, K_{t}^{+}, K_{t}^{-}\right)_{t \leq T}$ be the maximal solution of (3.6) then, for any $t \leq T$ we have

$$
Y_{t}=\eta+\int_{t}^{T} \tilde{F}\left(s, Y_{s}, Z_{s}\right) d s+\left(K_{T}^{+}-K_{t}^{+}\right)-\left(K_{T}^{-}-K_{t}^{-}\right)-\int_{t}^{T} Z_{s} d B_{s}
$$

Now for $t \leq T$ let us set

$$
\mathscr{y}_{t}=\frac{\operatorname{Ln} Y_{t}}{2 C}, \quad \mathscr{L}_{t}=\frac{Z_{t}}{2 C Y_{t}}, \quad d \mathscr{Y}_{t}^{ \pm}=\frac{d K_{t}^{ \pm}}{2 C Y_{t}} .
$$

These processes are well defined since $Y \geq \exp (2 \mathrm{Cm})$. By using Itô's formula with $\operatorname{Ln} Y / 2 C$ we obtain

$$
\mathscr{Y}_{t}=\xi+\int_{t}^{T} f\left(s, \mathscr{Y}_{s}, \mathscr{L}_{s}\right) d s+\left(\mathscr{K}_{T}^{+}-\mathscr{Y}_{t}^{+}\right)-\left(\mathscr{K}_{T}^{-}-\mathscr{K}_{t}^{-}\right)-\int_{t}^{T} \mathscr{L}_{s} d B_{s} .
$$


On the other hand, we have $Y \geq \exp (2 \mathrm{Cm})$ then $\mathscr{E} \in \mathscr{L}^{2, d}$ since $Z$ is so, and $\mathscr{K}^{ \pm} \in \mathcal{M}$ since $K^{ \pm}$belong also to $M$. In addition we obviously have $\int_{0}^{T}\left(\mathscr{Y}_{s}-L_{s}\right) d \mathcal{K}_{s}^{+}=\int_{0}^{T}\left(U_{s}-\right.$ $\left.\mathscr{Y}_{s}\right) d \mathcal{K}_{s}^{-}=0$ since $\int_{0}^{T}\left(Y_{s}-L_{s}^{\prime}\right) d K_{s}^{+}=\int_{0}^{T}\left(U_{s}^{\prime}-Y_{s}\right) d K_{s}^{-}=0$. Then the quadruple $\left(\mathscr{Y}, \mathscr{L}, \mathscr{K}^{+}\right.$, $\mathscr{K}^{-}$) satisfies $(2.1)$.

It remains to show that this solution is maximal. Let $\left(\overline{\mathscr{Y}}_{t}, \overline{\mathscr{Z}}_{t}, \overline{\mathcal{K}}_{t}^{+}, \overline{\mathscr{K}}_{t}^{-}\right)_{t \leq T}$ be another solution for (3.53), then

$$
\left(\exp \left(2 C \bar{y}_{t}\right), 2 C \exp \left(2 C \bar{y}_{t}\right) \mathscr{L}_{t}, \int_{0}^{t} 2 C \exp \left(2 C \bar{y}_{s}\right) d \overline{\mathscr{Y}}_{s}^{+}, \int_{0}^{t} 2 C \exp \left(2 C \overline{\bar{Y}_{s}}\right) d \overline{\mathscr{Y}}_{s}^{-}\right)_{t \leq T}
$$

is a solution for the reflected BSDE associated with $\left(F, \eta, L^{\prime}, U^{\prime}\right)$. Therefore we have $\exp \left(2 C_{\bar{y}}\right) \leq Y$ since $Y$ is a maximal solution and then $P$ - a.s., $\bar{Y} \leq \mathscr{Y}$.

Remark 3.3. A comparison result. Let $f$ and $f^{\prime}$ be two coefficients which satisfy (A2) and such that $P$ - a.s., $f(t, \omega, y, z) \leq f^{\prime}(t, \omega, y, z)$, for any $t, y$, and $z$. Let $\left(\mathscr{Y}_{t}, \mathscr{L}_{t}, \mathscr{K}_{t}^{+}, \mathscr{K}_{t}^{-}\right)_{t \leq T}$ (resp., $\left.\left(\mathscr{Y}_{t}^{\prime}, \mathscr{E}_{t}^{\prime}, \mathcal{K}_{t}^{\prime+}, \mathcal{K}_{t}^{\prime-}\right)_{t \leq T}\right)$ be the maximal solution of the double-barrier reflected BSDE associated with $(f, \xi, L, U)$ (resp., $\left.\left(f^{\prime}, \xi, L, U\right)\right)$, then $P$ - a.s , $\mathscr{y} \leq \mathcal{Y}^{\prime}$.

Actually the functions $f$ and $f^{\prime}$ satisfy (A2) with $C=\max \left\{C_{f}, C_{f^{\prime}}\right\}$. So let $F$ and $F^{\prime}$ be the functions defined as in (3.4) and associated with $f$ and $f^{\prime}$, respectively. Therefore we have $F \leq F^{\prime}$. Now let $\left(Y_{t}, Z_{t}, K_{t}^{+}, K_{t}^{-}\right)_{t \leq T}\left(\operatorname{resp} .,\left(Y_{t}^{\prime}, Z_{t}^{\prime}, K_{t}^{\prime+}, K_{t}^{\prime-}\right)_{t \leq T}\right)$ be the maximal solution of the double-barrier reflected BSDE associated with $\left(F, \eta, L^{\prime}, U^{\prime}\right)$ (resp., $\left.\left(F^{\prime}, \eta, L^{\prime}, U^{\prime}\right)\right)$, then we have $P$ - a.s., $Y \leq Y^{\prime}$. Henceforth from the definition we straightforwardly have $P$ - a.s., $Y \leq Y^{\prime}$.

\section{Connection with risk-sensitive mixed zero-sum game problem}

We now deal with an application of the double-barrier reflected BSDE to solve the mixed risk-sensitive zero-sum game problem. This problem consists in a system $\mathbf{S}$ on which two agents $c_{1}$ and $c_{2}$ intervene. The interventions of the agents have two forms, control and stopping, that is, they control $\mathbf{S}$ up to the time when one of them decides to stop controlling. Their actions are not free and their advantages are antagonistic, that is, there exits a payoff $J$, which depends on the implemented strategies, which is a reward for $c_{2}$ and a cost for $c_{1}$. On the other hand, the expression of $J$ integrates sensitiveness with respect to risk of the agents, either they are risk-seeking or risk-averse. Therefore the problem is to find two pairs of strategies for the agents which in a way are fair when implemented.

Let us describe precisely the setting of the problem. Let $\mathscr{C}$ be the set of continuous functions $\bar{w}$ from $[0, T]$ into $\mathbb{R}^{d}$ endowed with the uniform norm and let $\sigma$ be a function from $[0, T] \times \mathscr{C}$ into $\mathbb{R}^{d \times d}$ such that

(C1) $\sigma$ is $\mathscr{P}$-measurable, that is, for any continuous $\mathscr{P}$-measurable process $p=\left(p_{t}\right)_{t \leq T}$, the process $\left(\sigma\left(t, p_{t}\right)\right)_{t \leq T}$ is $\mathscr{P}$-measurable;

(C2) there exists a constant $k$ such that

(i) for all $t \in[0, T], \bar{w}, \bar{w}^{\prime} \in \mathscr{C},\left|\sigma(t, \bar{w})-\sigma\left(t, \bar{w}^{\prime}\right)\right| \leq k\left\|\bar{w}-\bar{w}^{\prime}\right\|_{t}$ where $\|\bar{w}\|_{t}=$ $\sup _{s \leq t}\left|\bar{w}_{s}\right|, t \leq T$;

(ii) for any $t \leq T,|\sigma(t, 0)| \leq k, \sigma$ is invertible and its inverse $\sigma^{-1}$ is bounded. 
These assumptions on $\sigma$ imply that the stochastic functional differential equation

$$
d x_{t}=\sigma(t, x, \cdot) d B_{t}, \quad t \leq T, x_{0}=\bar{x}_{0} \in \mathbb{R}^{d}
$$

has a unique solution $\left(x_{t}\right)_{t \leq T}$ (see, e.g., $[17,30]$ ). The process $x$ stands for the dynamic of the evolution of $\mathbf{S}$ when it is not controlled.

Let us now consider $U$ (resp., $V$ ) a compact metric space and $\mathcal{U}$ (resp., $\mathscr{V}$ ) the set of $\mathscr{P}$-measurable processes $u=\left(u_{t}\right)_{t \leq T}$ (resp., $v=\left(v_{t}\right)_{t \leq T}$ ) with values in $U$ (resp., $V$ ); $\boldsymbol{u}$ (resp., $\mathscr{V}$ ) represents the set of admissible controls for $c_{1}$ (resp., $c_{2}$ ). On the other hand, hereafter $\mathscr{U} \times \mathscr{V}$ is called the set of admissible controls for the agents.

Next let $f$ and $h$ be two measurable functions from $[0, T] \times \mathscr{C} \times U \times V$ into $\mathbb{R}^{d}$ and $\mathbb{R}^{+}$, respectively, such that

(C3) $f$ and $h$ are uniformly bounded and $\mathscr{P}$-measurable, that is, for any $u \in \mathcal{U}$ and $v \in \mathscr{V}$, the processes $f\left(t, x, u_{t}, v_{t}\right)_{t \leq T}$ and $h\left(t, x, u_{t}, v_{t}\right)_{t \leq T}$ are $\mathscr{P}$-measurable;

(C4) for all $t \in[0, T]$ the mapping $f(t, x, \cdot, \cdot)$ (resp., $h(t, x, \cdot \cdot \cdot))$ which with $(u, v)$ in $U \times V$ associates $f(t, x, u, v)$ (resp., $h(t, x, u, v))$ is continuous.

Now let $(u, v) \in \mathscr{U} \times \mathscr{V}$ and $P^{u, v}$ be the probability on $(\Omega, \mathscr{F})$ defined by

$$
\frac{d P^{u, v}}{d P}=\exp \left\{\int_{0}^{T} \sigma^{-1}(s, x) f\left(s, x, u_{s}, v_{s}\right) d B_{s}-\frac{1}{2} \int_{0}^{T}\left|\sigma^{-1}(s, x) f\left(s, x, u_{s}, v_{s}\right)\right|^{2} d s\right\} .
$$

So according to Girsanov's theorem (see, e.g., $[17,30])$, for any $(u, v) \in \mathscr{U} \times \mathscr{V}$, the process $B^{u, v}:=\left(B_{t}-\int_{0}^{t} \sigma^{-1}(s, x) f\left(s, x, u_{s}, v_{s}\right) d s\right)_{t \leq T}$ is a Brownian motion on $\left(\Omega, \mathscr{F}, P^{u, v}\right)$ and $x$ is a weak solution of the following functional differential equation:

$$
d x_{t}=f\left(t, x, u_{t}, v_{t}\right) d t+\sigma(t, x) d B_{t}^{u, v}, \quad t \leq T, x_{0}=\bar{x}
$$

When the agent $c_{1}$ (resp., $c_{2}$ ) acts on $\mathbf{S}$ with a control $u$ (resp., $v$ ), its dynamic of evolution has the same law as the one of $x$ under $P^{u, v}$. In a way, this means that the interventions of the agents generate a drift.

Assume now that $c_{1}$ (resp., $c_{2}$ ) decides, on the one hand, to use the control $u$ (resp., $v$ ) and, on the other hand, to stop controlling at a stopping time $\tau$ (resp., $\nu$ ). The payment between the two agents, which is a cost for $c_{1}$ and a reward for $c_{2}$, is given by

$$
J(u, \tau ; v, \nu)=E^{u, v}\left[\exp \theta\left\{\int_{0}^{v \wedge \tau} h\left(s, x, u_{s}, v_{s}\right) d s+L_{\nu} 1_{[\nu \leq \tau<T]}+U_{\tau} 1_{[\tau<\nu]}+\xi 1_{[\tau=\nu=T]}\right\}\right],
$$

where $L, U$, and $\xi$ are those of the previous section. The constant $\theta$ is related to sensitiveness of the controllers with respect to risk. In the case when they are risk-seeking (resp., risk-averse), $\theta>0$ (resp., <0). Hereafter without loss of generality, we assume $\theta=1$. The objective of $c_{1}$ (resp., $c_{2}$ ) is to minimize (resp., maximize) the payoff $J(u, \tau ; v, v)$. Therefore the problem is to find a strategy $\left(u^{*}, \tau^{*} ; v^{*}, v^{*}\right) \in \mathcal{U} \times \mathscr{T} \times \mathscr{V} \times \mathscr{T}$ such that for any $(u, \tau)$ 
and $(v, v)$ we have

$$
J\left(u^{*}, \tau^{*} ; v, v\right) \leq J\left(u^{*}, \tau^{*} ; v^{*}, v^{*}\right) \leq J\left(u, \tau ; v^{*}, v^{*}\right) .
$$

This strategy is called a saddle-point for the controllers. The inequalities (4.5) show that $\left(u^{*}, \tau^{*} ; v^{*}, v^{*}\right)$ is actually fair since when $c_{1}$ (resp., $\left.c_{2}\right)$ decides to act on $\mathbf{S}$ with $\left(u^{*}, \tau^{*}\right)$ (resp., $\left.\left(v^{*}, v^{*}\right)\right)$, then the best that $c_{2}$ (resp., $\left.c_{1}\right)$ has to do is to act with $\left(v^{*}, v^{*}\right)$ (resp., $\left.\left(u^{*}, \tau^{*}\right)\right)$, otherwise he will earn (resp., pay) less (resp., more).

The expression of $J(u, \tau ; v, v)$ can be understood in the following way:

(i) $h\left(t, x, u_{t}, v_{t}\right)$ is the instantaneous cost (resp., reward) for $c_{1}$ (resp., $c_{2}$ );

(ii) $L_{\nu}$ is the reward (resp., cost) for $c_{2}$ (resp., $c_{1}$ ) if $c_{2}$ decides first to stop controlling at $v$;

(iii) $U_{\tau}$ is the cost (resp., reward) for $c_{1}$ (resp., $c_{2}$ ) if $c_{1}$ decides first to stop controlling at $\tau$.

Now let $H$ be the Hamiltonian function associated with this game problem, that is, the function which with $(t, x, p, u, v) \in[0, T] \times \mathscr{b} \times \mathbb{R}^{d} \times U \times V$ associates

$$
H(t, x, p, u, v):=p \sigma^{-1}(t, x) f(t, x, u, v)+h(t, x, u, v)
$$

Hereafter we suppose Isaacs condition fulfilled, that is,

$$
\text { [H1]: } \inf _{u \in U} \sup _{v \in V} H(t, x, p, u, v)=\sup _{v \in V} \inf _{u \in U} H(t, x, p, u, v), \quad P \text {-a.s. }
$$

Under [H1], through Benes'selection theorem [2], there exists a couple of $\mathscr{P} \times \mathscr{B}\left(\mathbb{R}^{d}\right)$ measurable functions $u^{*}(t, x, p)$ and $v^{*}(t, x, p)$ with values respectively in $U$ and $V$ such that $P$ - a.s., for any $(t, u, p) \in[0, T] \times \mathscr{C} \times \mathbb{R}^{d}, u \in U$, and $v \in V$,

$$
H\left(t, x, p, u^{*}(t, x, p), v^{*}(t, x, p)\right)=\inf _{u \in U} \sup _{v \in V} H(t, x, p, u, v)=\sup _{v \in V} \inf _{u \in U} H(t, x, p, u, v) .
$$

In addition (see, e.g., [4] for that fact) we have

$$
H\left(t, x, p, u^{*}(t, x, p), v\right) \leq H\left(t, x, p, u^{*}(t, x, p), v^{*}(t, x, p)\right) \leq H\left(t, x, p, u, v^{*}(t, x, p)\right) .
$$

Let us now consider the function $H^{*}$ which with $(t, x, p) \in[0, T] \times \mathscr{C} \times \mathbb{R}^{d}$ associates:

$$
H^{*}(t, x, p)=H^{*}\left(t, x, p, u^{*}(t, x, p), v^{*}(t, x, p)\right)
$$

The function $p \mapsto H^{*}(t, x, p)+(1 / 2)|p|^{2}$ is continuous and at most with quadratic growth with respect to $p$ since $f, h$, and $\sigma^{-1}$ are bounded. The following theorem is the main result of this section. 
Theorem 4.1. Assume that [H1] holds true and let $\left(Y^{*}, Z^{*}, K^{*,+}, K^{*,-}\right)$ be the maximal solution of the following double barrier reflected BSDE:

$$
\begin{gathered}
Y^{*} \in \mathscr{S}^{2}, \quad Z^{*} \in \mathscr{L}^{2, d}, \quad K^{*,+}, K^{*,-} \in \mathcal{M} \\
Y_{t}^{*}=\xi+\int_{t}^{T}\left\{H^{*}\left(s, x, Z_{s}^{*}\right)+\frac{1}{2}\left|Z_{s}^{*}\right|^{2}\right\} d s+\left(K_{T}^{*,+}-K_{t}^{*,+}\right)-\left(K_{T}^{*,-}-K_{t}^{*,-}\right)-\int_{t}^{T} Z_{s}^{*} d B_{s}, \\
L_{t} \leq Y_{t} \leq U_{t}, \quad \forall t \leq T, \quad \int_{t}^{T}\left(Y_{s}^{*}-L_{s}\right) d K_{s}^{*,+}=\int_{t}^{T}\left(U_{s}-Y_{s}^{*}\right) d K_{s}^{*,-}=0 .
\end{gathered}
$$

Set $u^{*}:=\left(u^{*}\left(t, x, Z_{t}^{*}\right)\right)_{t \leq T}, v^{*}:=\left(v^{*}\left(t, x, Z_{t}^{*}\right)\right)_{t \leq T}, v^{*}:=\inf \left\{t \in[0, T], Y_{t}^{*}=L_{t}\right\} \wedge T$, and finally $\tau^{*}:=\inf \left\{t \in[0, T], Y_{t}^{*}=U_{t}\right\} \wedge T$, then $\exp \left(Y_{0}^{*}\right)=J\left(u^{*}, \tau^{*} ; v^{*}, v^{*}\right)$. In addition $\left(u^{*}, \tau^{*} ; v^{*}, v^{*}\right)$ is a saddle-point strategy for the risk-sensitive mixed zero-sum differential game.

Proof. According to Theorem 3.2, the double-barrier reflected BSDE associated with $\left(H^{*}(t, x, z)+(1 / 2)|z|^{2}, \xi, L, U\right)$ has a maximal solution $\left(Y_{t}^{*}, Z_{t}^{*}, K_{t}^{*,+}, K_{t}^{*,-}\right)_{t \leq T}$. Then for any $t \leq T$ we have

$$
Y_{t}^{*}=\xi+\int_{t}^{T}\left[H^{*}\left(s, x, Z_{s}^{*}\right)+\frac{1}{2}\left|Z_{s}^{*}\right|^{2}\right] d s+K_{T}^{*,+}-K_{t}^{*,+}-\left(K_{T}^{*,-}-K_{t}^{*,-}\right)-\int_{t}^{T} Z_{s}^{*} d B_{s} .
$$

For $t \leq T$ let us set $\bar{Y}_{t}^{*}=\exp \left(Y_{t}^{*}\right)$. Using Itô's formula we obtain

$$
\bar{Y}_{t}^{*}=\exp (\xi)+\int_{t}^{T} \bar{Y}_{s}^{*} H^{*}\left(s, x, Z_{s}^{*}\right) d s+\int_{t}^{T} \bar{Y}_{s}^{*} d K_{s}^{*,+}-\int_{t}^{T} \bar{Y}_{s}^{*} d K_{s}^{*,-}-\int_{t}^{T} \bar{Y}_{s}^{*} Z_{s}^{*} d B_{s}
$$

Then

$$
\bar{Y}_{t}^{*}=\exp (\xi)+\int_{t}^{T} \bar{Y}_{s}^{*} h^{*}\left(s, x, Z_{s}^{*}\right) d s+\int_{t}^{T} \bar{Y}_{s}^{*} d K_{s}^{*,+}-\int_{t}^{T} \bar{Y}_{s}^{*} d K_{s}^{*,-}-\int_{t}^{T} \bar{Y}_{s}^{*} Z_{s}^{*} d B_{s}^{u^{*}, v^{*}}
$$

where $h^{*}\left(s, x, Z_{s}^{*}\right)=h\left(s, x,\left(u^{*}, v^{*}\right)\left(s, x, Z_{s}^{*}\right)\right)$. Now let us set $\tilde{Y}_{t}^{*}=\bar{Y}_{t}^{*} \exp \left\{\int_{o}^{t} h^{*}(s, x\right.$, $\left.\left.Z_{s}^{*}\right) d s\right\}, t \leq T$, then $\tilde{Y}$ is bounded since $\bar{Y}^{*}$ and $h$ are so. On the other hand Itô's formula implies

$$
-d \tilde{Y}_{t}^{*}=\tilde{Y}_{t}^{*} d K_{t}^{*,+}-\tilde{Y}_{t}^{*} d K_{t}^{*,-}-\tilde{Y}_{t}^{*} Z_{t}^{*} d B_{t}^{u^{*}, v^{*}}, \quad t \leq T .
$$


Now for $k \geq 0$ let $\tau_{k}=\inf \left\{t \geq 0, \int_{0}^{t}\left|Z_{s}^{*}\right|^{2} d s \geq k\right\} \wedge T$, therefore $\tau_{k}$ is a stopping time such that $\tau_{k} \uparrow T$ as $k \rightarrow \infty$. On the other hand $\tilde{Y}_{0}^{*}$ is $F_{0}$-measurable, then it is equal to a constant, therefore $\tilde{Y}_{0}^{*}=E^{u^{*}, v^{*}}\left[\tilde{Y}_{0}^{*}\right]$. In addition we have,

$$
\begin{aligned}
\tilde{Y}_{0}^{*}=E^{u^{*}, v^{*}}[ & \tilde{Y}_{\tau^{*} \wedge v^{*} \wedge \tau_{k}}^{*}+\int_{0}^{\tau^{*} \wedge v^{*} \wedge \tau_{k}} \tilde{Y}_{s}^{*} d K_{s}^{*,+}-\int_{t}^{\tau^{*} \wedge v^{*} \wedge \tau_{k}} \tilde{Y}_{s}^{*} d K_{s}^{*,-} \\
& \left.-\int_{0}^{\tau^{*} \wedge v^{*} \wedge \tau_{k}} \tilde{Y}_{s}^{*} Z_{s}^{*} d B_{s}^{u^{*}, v^{*}}\right] .
\end{aligned}
$$

The process $K^{*,+}$ (resp., $K^{*,-}$ ) is increasing only when $Y^{*}=L$ (resp., $Y^{*}=U$ ), then it does not increase between 0 and $\tau^{*} \wedge \nu^{*}$. It follows that $\int_{0}^{\tau^{*} \wedge v^{*}}\left(\tilde{Y}_{s}^{*}-L_{s}\right) d K_{s}^{*,+}=0$ (resp., $\left.\int_{0}^{\tau^{*} \wedge v^{*}}\left(\tilde{Y}_{s}^{*}-U_{s}\right) d K_{s}^{*,-}=0\right)$.

On the other hand, the process $\left(\int_{0}^{t \wedge \tau_{k}} \tilde{Y}_{s}^{*} Z_{s}^{*} d B_{s}^{u^{*}, v^{*}}\right)_{t \leq T}$ is a $P^{u^{*}, v^{*}}$-martingale. Indeed by the Burkholder-Davis-Gundy inequality we obtain

$$
\begin{aligned}
E^{u^{*}, v^{*}}\left[\sup _{t \leq \tau_{k}}\left|\int_{0}^{t} \tilde{Y}_{s}^{*} Z_{s}^{*} d B_{s}^{u^{*}, v^{*}}\right|\right] & \leq C E^{u^{*}, v^{*}}\left[\sqrt{\int_{0}^{\tau_{k}}\left|\tilde{Y}_{s}^{*}\right|^{2}\left|Z_{s}^{*}\right|^{2} d s}\right] \\
& \leq C^{\prime} \sqrt{E\left[\left(\frac{d P^{u^{*}, v^{*}}}{d P}\right)^{2}\right]} \sqrt{E\left[\int_{0}^{\tau_{k}}\left|Z_{s}^{*}\right|^{2} d s\right]}<\infty
\end{aligned}
$$

since $\tilde{Y}^{*}, \sigma^{-1}$, and $f$ are bounded. Then for any $k \geq 0$, we have $\tilde{Y}_{0}^{*}=E^{u^{*}, v^{*}}\left[\tilde{Y}_{\tau^{*} \wedge v^{*} \wedge \tau_{k}}^{*}\right]$. But the process $\tilde{Y}^{*}$ is bounded, then from Lebesgue's dominated convergence theorem we obtain

$$
\begin{aligned}
\tilde{Y}_{0}^{*} & =E^{u^{*}, v^{*}}\left[\tilde{Y}_{\tau^{*} \wedge \nu^{*}}^{*}\right]=E^{u^{*}, v^{*}}\left[\exp \left(\int_{0}^{\tau^{*} \wedge v^{*}} h^{*}\left(s, x, Z_{s}^{*}\right) d s+Y_{\tau^{*} \wedge \nu^{*}}^{*}\right)\right] \\
& =E^{u^{*}, v^{*}}\left[\exp \left\{\int_{0}^{\tau^{*} \wedge v^{*}} h^{*}\left(s, x, Z_{s}^{*}\right) d s+\xi 1_{\left[\tau^{*}=v^{*}=T\right]}+L_{\nu^{*}} 1_{\left[\nu^{*} \leq \tau^{*}<T\right]}+U_{\tau^{*}} 1_{\left[\tau^{*}<v^{*}\right]}\right\}\right] .
\end{aligned}
$$

Henceforth

$$
\exp \left(Y_{0}^{*}\right)=J\left(u^{*}, \tau^{*} ; v^{*}, v^{*}\right)
$$

Now, let us consider $u \in \mathcal{U}$ and $v \in \mathcal{T}$. Let $\left(Y_{t}^{u}, Z_{t}^{u}\right)_{t \leq T}$ be the solution of the following BSDE: for all $t \leq T$,

$$
\begin{aligned}
Y_{t \wedge \tau \wedge \nu^{*}}^{u}= & \int_{t \wedge \tau \wedge v^{*}}^{\tau \wedge \nu^{*}}\left\{H\left(s, x, Z_{s}^{u}, u_{s}, v^{*}\left(s, x, Z_{s}^{*}\right)\right)+\frac{1}{2}\left|Z_{s}^{u}\right|^{2}\right\} d s-\int_{t \wedge \tau \wedge \nu^{*}}^{\tau \wedge v^{*}} Z_{s}^{u} d B_{s} \\
& +\xi 1_{\left[\tau=v^{*}=T\right]}+U_{\tau} 1_{\left[\tau<\nu^{*}\right]}+L_{\gamma^{*}} 1_{\left[\nu^{*} \leq \tau<T\right]} .
\end{aligned}
$$


There is no problem of existence of $\left(Y_{t}^{u}, Z_{t}^{u}\right)_{t \leq T}$ since $\xi 1_{\left[\tau=\nu^{*}=T\right]}+U_{\tau} 1_{\left[\tau<\nu^{*}\right]}+L_{\nu^{*}} 1_{\left[\nu^{*} \leq \tau<T\right]}$ is $F_{T}$-measurable, bounded and $H\left(t, x, z, u_{t}, v^{*}\left(t, x, Z_{t}^{*}\right)\right)+(1 / 2)|z|^{2}$ is continuous with quadratic growth with respect to $z$ (see, e.g., $[19,23]$ ). On the other hand we have

$$
Y_{t \wedge \tau \wedge \nu^{*}}^{*}=Y_{\tau \wedge \nu^{*}}^{*}+\int_{t \wedge \tau \wedge \nu^{*}}^{\tau \wedge \nu^{*}}\left\{H^{*}\left(s, x, Z_{s}^{*}\right)+\frac{1}{2}\left|Z_{s}^{*}\right|^{2}\right\} d s-\int_{t \wedge \tau \wedge v^{*}}^{\tau \wedge \nu^{*}} d K_{s}^{*,-}-\int_{t \wedge \tau \wedge v^{*}}^{\tau \wedge \nu^{*}} Z_{s}^{*} d B_{s}
$$

Now for $t \leq T$, let $\bar{Y}_{t}^{u}=\exp \left(Y_{t}^{u}\right)$. Using Itô's formula we obtain

$$
\begin{aligned}
\left(\bar{Y}_{t \wedge \tau \wedge \nu^{*}}^{*}-\bar{Y}_{t \wedge \tau \wedge \nu^{*}}^{u}\right)= & \exp \left(Y_{\tau \wedge \nu^{*}}^{*}\right)-\exp \left(\xi 1_{\left[\tau=v^{*}=T\right]}+U_{\tau} 1_{\left[\tau<\nu^{*}\right]}+L_{\gamma^{*}} 1_{\left[\nu^{*} \leq \tau<T\right]}\right) \\
& +\int_{t \wedge \tau \wedge \nu^{*}}^{\tau \wedge \nu^{*}} \bar{Y}_{s}^{*} H^{*}\left(s, x, Z_{s}^{*}\right) d s \\
& -\int_{t \wedge \tau \wedge \nu^{*}}^{\tau \wedge \nu^{*}} \bar{Y}_{s}^{u} H\left(s, x, Z_{s}^{u}, u_{s}, v^{*}\left(s, x, Z_{s}^{*}\right)\right) d s \\
& -\int_{t \wedge \tau \wedge \nu^{*}}^{\tau \wedge \nu^{*}}\left(\bar{Y}_{s}^{*} Z_{s}^{*}-\bar{Y}_{s}^{u} Z_{s}^{u}\right) d B_{s}-\int_{t \wedge \tau \wedge \nu^{*}}^{\tau \wedge \nu^{*}} \bar{Y}_{s}^{*} d K_{s}^{*,-}, \quad t \leq T .
\end{aligned}
$$

Then

$$
\begin{aligned}
& \left(\bar{Y}_{t \wedge \tau \wedge \nu^{*}}^{*}-\bar{Y}_{t \wedge \tau \wedge \nu^{*}}^{u}\right)=\exp \left(Y_{\tau \wedge \nu^{*}}^{*}\right)-\exp \left(\xi 1_{\left[\tau=v^{*}=T\right]}+U_{\tau} 1_{\left[\tau<\nu^{*}\right]}+L_{\nu^{*}} 1_{\left[\nu^{*}<\tau \leq T\right]}\right) \\
& +\int_{t \wedge \tau \wedge v^{*}}^{\tau \wedge \nu^{*}} \bar{Y}_{s}^{*}\left\{H^{*}\left(s, x, Z_{s}^{*}\right)-H\left(s, x, Z_{s}^{*}, u_{s}, v^{*}\left(s, x, Z_{s}^{*}\right)\right)\right\} d s \\
& +\int_{t \wedge \tau \wedge v^{*}}^{\tau \wedge v^{*}}\left\{\bar{Y}_{s}^{*} H\left(s, x, Z_{s}^{*}, u_{s}, v^{*}\left(s, x, Z_{s}^{*}\right)\right)\right. \\
& \left.-\bar{Y}_{s}^{u} H\left(s, x, Z_{s}^{u}, u_{s}, v^{*}\left(s, x, Z_{s}^{*}\right)\right)\right\} d s \\
& -\int_{t \wedge \tau \wedge v^{*}}^{\tau \wedge v^{*}}\left(\bar{Y}_{s}^{*} Z_{s}^{*}-\bar{Y}_{s}^{u} Z_{s}^{u}\right) d B_{s}-\int_{t \wedge \tau \wedge v^{*}}^{\tau \wedge v^{*}} \bar{Y}_{s}^{*} d K_{s}^{*,-} \\
& =\exp \left(Y_{\tau \wedge \nu^{*}}^{*}\right)-\exp \left(\xi 1_{\left[\tau=\nu^{*}=T\right]}+U_{\tau} 1_{\left[\tau<\nu^{*}\right]}+L_{\gamma^{*}} 1_{\left[\nu^{*} \leq \tau<T\right]}\right) \\
& +\int_{t \wedge \tau \wedge v^{*}}^{\tau \wedge v^{*}} \bar{Y}_{s}^{*}\left\{H^{*}\left(s, x, Z_{s}^{*}\right)-H\left(s, x, Z_{s}^{*}, u_{s}, v^{*}\left(s, x, Z_{s}^{*}\right)\right)\right\} d s \\
& +\int_{t \wedge \tau \wedge v^{*}}^{\tau \wedge v^{*}}\left(\bar{Y}_{s}^{*}-\bar{Y}_{s}^{u}\right) h\left(s, x, u_{s}, v^{*}\left(s, x, Z_{s}^{*}\right)\right) d s \\
& -\int_{t \wedge \tau \wedge v^{*}}^{\tau \wedge v^{*}}\left(\bar{Y}_{s}^{*} Z_{s}^{*}-\bar{Y}_{s}^{u} Z_{s}^{u}\right) d B_{s}^{u, v^{*}\left(s, x, Z_{s}^{*}\right)}-\int_{t \wedge \tau \wedge v^{*}}^{\tau \wedge \nu^{*}} \bar{Y}_{s}^{*} d K_{s}^{*,-} .
\end{aligned}
$$


Now let us set $D_{t}=\left(\bar{Y}_{t}^{*}-\bar{Y}_{t}^{u}\right) \exp \left(\int_{0}^{t} h\left(s, x, u_{s}, v^{*}\left(s, x, Z_{s}^{*}\right)\right) d s\right), t \leq T$. Then we have for all $t \leq T$,

$$
\begin{aligned}
D_{t \wedge \tau \wedge v^{*}}= & \int_{t \wedge \tau \wedge v^{*}}^{\tau \wedge v^{*}} \exp \left(\int_{0}^{s} h\left(r, x, u_{s}, v^{*}\left(r, x, Z_{r}^{*}\right)\right) d r\right) \bar{Y}_{s}^{*} \\
& \times\left\{H^{*}\left(s, x, Z_{s}^{*}\right)-H\left(s, x, Z_{s}^{*}, u_{s}, v^{*}\left(s, x, Z_{s}^{*}\right)\right)\right\} d s \\
& -\int_{t \wedge \tau \wedge v^{*}}^{\tau \wedge \nu^{*}} \exp \left(\int_{0}^{s} h\left(r, x, u_{s}, v^{*}\left(r, x, Z_{r}^{*}\right)\right) d r\right)\left(\bar{Y}_{s}^{*} Z_{s}^{*}-\bar{Y}_{s}^{u} Z_{s}^{u}\right) d B_{s}^{u, v^{*}\left(s, x, Z_{s}^{*}\right)} \\
& -\int_{t \wedge \tau \wedge \nu^{*}}^{\tau \wedge v^{*}} \exp \left(\int_{0}^{s} h\left(r, x, u_{s}, v^{*}\left(r, x, Z_{r}^{*}\right)\right) d r\right) \bar{Y}_{s}^{*} d K_{s}^{*,-}+D_{\tau \wedge v^{*}}
\end{aligned}
$$

Next for $n \geq 0$, let $\tau_{n}$ be the stopping time defined by $\tau_{n}=\inf \left\{t \geq 0, \int_{0}^{t}\left(\left|Z_{s}^{*}\right|^{2}+\left|Z_{s}^{u}\right|^{2}\right) d s \geq\right.$ $n\} \wedge T$. Then $\tau_{n}>T$ as $n \rightarrow \infty$ and we have

$$
\begin{aligned}
D_{t \wedge \tau \wedge \nu^{*} \wedge \tau_{n}=} & D_{\tau \wedge \nu^{*} \wedge \tau_{n}}+\int_{t \wedge \tau \wedge \nu^{*} \wedge \tau_{n}}^{\tau \wedge \nu^{*} \wedge \tau_{n}} \exp \left(\int_{0}^{s} h\left(r, x, u_{r}, v^{*}\left(r, x, Z_{r}^{*}\right)\right) d r\right) \bar{Y}_{s}^{*} \\
& \times\left\{H^{*}\left(s, x, Z_{s}^{*}\right)-H\left(s, x, Z_{s}^{*}, u_{s}, v^{*}\left(s, x, Z_{s}^{*}\right)\right)\right\} d s \\
& -\int_{t \wedge \tau \wedge \nu^{*} \wedge \tau_{n}}^{\tau \wedge \nu^{*} \wedge \tau_{n}} \exp \left(\int_{0}^{s} h\left(r, x, u_{r}, v^{*}\left(r, x, Z_{r}^{*}\right)\right) d r\right)\left(\bar{Y}_{s}^{*} Z_{s}^{*}-\bar{Y}_{s}^{u} Z_{s}^{u}\right) d B_{s}^{u, v^{*}\left(s, x, Z_{s}^{*}\right)} \\
& -\int_{t \wedge \tau \wedge v^{*} \wedge \tau_{n}}^{\tau \wedge \nu^{*} \wedge \tau_{n}} \exp \left(\int_{0}^{s} h\left(r, x, u_{r}, v^{*}\left(r, x, Z_{r}^{*}\right)\right) d r\right) \bar{Y}_{s}^{*} d K_{s}^{*,-} .
\end{aligned}
$$

As $\bar{Y}^{*} \geq 0, d K^{*-} \geq 0$, and $H^{*}\left(t, x, Z_{t}^{*}\right)-H\left(t, x, Z_{t}^{*}, u_{t}, v^{*}\left(t, x, Z_{t}^{*}\right)\right) \leq 0$, then taking the conditional expectation in (4.25) yields $D_{t \wedge \tau \wedge \nu^{*} \wedge \tau_{n}} \leq E^{u, v^{*}\left(t, x, Z_{t}^{*}\right)}\left[D_{\tau \wedge \nu^{*} \wedge \tau_{n}} \mid F_{t \wedge \tau \wedge \nu^{*} \wedge \tau_{n}}\right]$ for any $t \leq T$. But $\bar{Y}_{\tau \wedge \nu^{*}}^{*} \leq \bar{Y}_{\tau \wedge \nu^{*}}^{u}$, then $D_{\tau \wedge \nu^{*}} \leq 0$. Therefore if we use the dominated convergence theorem we obtain that, for any $t \leq T, D_{t \wedge \tau \wedge \nu^{*}} \leq 0$ since for any $t \leq T$, $\left(E^{u, v^{*}}\left[D_{\tau \wedge \nu^{*} \wedge \tau_{n}} \mid F_{t \wedge \tau \wedge \nu^{*} \wedge \tau_{n}}\right]\right)_{n \geq 0} \rightarrow E^{u, v^{*}}\left[D_{\tau \wedge \nu^{*}} \mid F_{t \wedge \tau \wedge \nu^{*}}\right]$ in $L^{1}\left(d P^{u, v^{*}}\right)$ as $n \rightarrow \infty$. Finally $D_{0} \leq 0$ implies that

$$
\exp \left(Y_{0}^{*}\right) \leq \exp \left(Y_{0}^{u}\right)
$$

On the other hand we have

$$
J\left(u, \tau ; v^{*}, v^{*}\right)=\exp \left(Y_{0}^{u}\right)
$$

Indeed,

$$
\bar{Y}_{0}^{u}=\bar{Y}_{\tau \wedge v^{*}}^{u}+\int_{0}^{\tau \wedge v^{*}} \bar{Y}_{s}^{u} H\left(s, Z_{s}^{u}, u_{s}, v^{*}\left(s, x, Z_{s}^{*}\right)\right) d s-\int_{0}^{\tau \wedge v^{*}} \bar{Y}_{s}^{u} Z_{s}^{u} d B_{s}
$$


Let us set $\tilde{Y}_{t}^{u}=\exp \left(\int_{0}^{t} h\left(s, x, u_{s}, v^{*}\left(s, x, Z_{s}^{*}\right)\right) d s\right) \bar{Y}_{t}^{u}, t \leq T ; \tilde{Y}^{u}$ is bounded since $h$ and $\bar{Y}^{u}$ are also bounded. On the other hand, Itô's formula implies that for any $t \leq \tau \wedge \nu^{*}$,

$$
d \tilde{Y}_{t}^{u}=\tilde{Y}_{t}^{u} Z_{t}^{u} d B_{t}^{u, v^{*}\left(t, x, Z_{t}^{*}\right)}
$$

Now for $n \geq 0$ let $\delta_{n}=\inf \left\{t \geq 0, \int_{0}^{t}\left|Z_{s}^{u}\right|^{2} \geq n\right\} \wedge T$, therefore $\delta_{n}$ is a stopping time such that $\delta_{n} \nearrow T$ as $n \rightarrow \infty$. Then according to (4.29) we obtain

$$
\begin{aligned}
\tilde{Y}_{0}^{u} & =E^{u, v^{*}\left(t, x, Z_{t}^{*}\right)}\left[\tilde{Y}_{0}^{u}\right] \\
& =E^{u, v^{*}\left(t, x, Z_{t}^{*}\right)}\left[\tilde{Y}_{\tau \wedge v^{*} \wedge \delta_{n}}^{u}-\int_{0}^{\tau \wedge v^{*} \wedge \delta_{n}} \tilde{Y}_{s}^{u} Z_{s}^{u} d B_{s}^{u, v^{*}\left(s, x, Z_{s}^{*}\right)}\right] \\
& =E^{u, v^{*}\left(t, x, Z_{t}^{*}\right)}\left[\tilde{Y}_{\tau \wedge v^{*} \wedge \delta_{n}}^{u}\right],
\end{aligned}
$$

since $\int_{0}^{t \wedge \delta_{n}} \tilde{Y}_{s}^{u} Z_{s}^{u} d B_{s}^{u, v^{*}\left(s, x, Z_{s}^{*}\right)}$ is a $P^{u, v^{*}\left(x, t, Z_{t}^{*}\right)}$-martingale. But the process $\tilde{Y}^{u}$ is bounded, then using the Lebesgue-dominated convergence theorem we obtain

$$
\begin{aligned}
& \tilde{Y}_{0}^{u}=E^{u, v^{*}\left(t, x, Z_{t}^{*}\right)}\left[\tilde{Y}_{\tau \wedge v^{*}}^{u}\right] \\
&=E^{u, v^{*}\left(t, x, Z_{t}^{*}\right)}\left[\exp \left\{\int_{0}^{\tau \wedge v^{*}} h\left(s, x, u_{s}, v^{*}\left(s, x, Z_{s}^{*}\right)\right) d s+Y_{\tau \wedge \nu^{*}}^{u}\right\}\right] \\
&=E^{u, v^{*}\left(t, x, Z_{t}^{*}\right)}\left[\operatorname { e x p } \left\{\int_{0}^{\tau \wedge v^{*}} h\left(s, x, u_{s}, v^{*}\left(s, x, Z_{s}^{*}\right)\right) d s\right.\right. \\
&\left.\left.\quad+\xi 1_{\left[\tau=v^{*}=T\right]}+U_{\tau} 1_{\left[\tau<\nu^{*}\right]}+L_{\nu^{*}} 1_{\left[v^{*} \leq \tau<T\right]}\right\}\right] \\
&=J\left(u, \tau, ; v, v^{*}\right) .
\end{aligned}
$$

Henceforth, taking into account (4.19) and (4.26), we obtain

$$
J\left(u^{*}, \tau^{*} ; v^{*}, v^{*}\right) \leq J\left(u, \tau ; v^{*}, v^{*}\right) .
$$

In the same way we can show that for any $v \in \mathcal{U}$ and $v \in \mathcal{T}$ we have

$$
J\left(u^{*}, \tau^{*} ; v, v\right) \leq J\left(u^{*}, \tau^{*} ; v^{*}, v^{*}\right)
$$

Thus we showed that $\left(u^{*}, \tau^{*} ; v^{*}, v^{*}\right)$ is a saddle-point for the mixed zero-sum sensitive game.

Remark 4.2. The process $\left(\exp \left(Y_{t}^{*}\right)\right)_{t \leq T}$ is the value function of the risk-sensitive mixed zero-sum differential game. This characterization combined with the fact that in the 


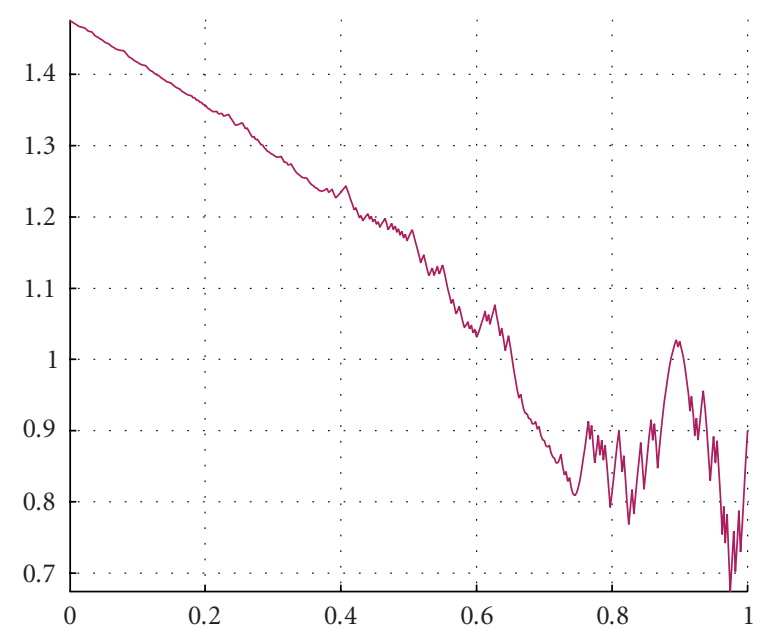

Figure 4.1. Simulation of a path of $Y\left(Y_{0}=1.4755\right)$.

previous proof the maximality of the solution has not been used, imply that the solution of the BSDE associated with $\left(H^{*}(t, x, z)+(1 / 2)|z|^{2}, \xi, L, U\right)$ is unique.

We are now going to deal with an example for which we give also a numerical treatment. The simulations are made on the ground of a paper by Mémin et al. [24]. Basically in that work, the theoretical BSDE is associated with a discrete BSDE where the Brownian motion is replaced by a weighted sum of independent Bernoulli symmetric random variables. Then the discrete BSDE is solved backwardly. Finally with that solution, a process is constructed which converges uniformly to the theoretical solution of the BSDE.

Example 4.3. Assume that $T=1$ and $d=1$ and let us set $L_{t}=0.5 \wedge\left|B_{t}\right|, U_{t}=\max \{3$, $\left.\left|B_{t}\right| \wedge 2\right\}$ for any $t \leq 1$ and $\xi=\left|B_{1}\right| \wedge 1$. On the other hand, let $x=\left(x_{t}\right)_{t \leq T}$ be the process such that for any $t \leq 1, x_{t}=\bar{x}+B_{t}$.

Now let $U=[0,1]$, let $V=[-1,1]$, and let $f$ (resp., $h$ ) be the function defined by $f(t, x, u, v)=u+2 v$ (resp., $\left.h(t, x, u, v)=(1 / 2)\left(u^{2}+v^{2}\right)\right)$. Obviously in that case Isaacs condition is satisfied and the function $H^{*}$ of $(4.8)$ is given by

$$
\begin{aligned}
H^{*}(t, x, p) & =H\left(t, x, p, u^{*}(t, x, p), v^{*}(t, x, p)\right) \\
& =\frac{4 p+1}{2} 1_{[p \geq 0]}+\frac{1-4 p-p^{2}}{2} 1_{[-1 \leq p<0]}+\frac{2-2 p}{2} 1_{[p<-1]}
\end{aligned}
$$

with $u^{*}(t, x, p)=1_{[p<-1]}-p 1_{[-1 \leq p<0]}$ and $v^{*}(t, x, p)=1_{[p \geq 0]}-1_{[p<0]}$.

Let $\left(Y_{t}, Z_{t}, K_{t}^{+}, K_{t}^{-}\right)_{t \leq 1}$ be the solution of the double-barrier reflected BSDE associated with $\left(H^{*}\left(t, x_{t}, z\right)+(1 / 2)|z|^{2}, \xi, L, U\right)$. The following drawing is a simulation of a path of $Y$.

Figure 4.1 shows the simulation of a path of $Y$. 


\section{Application in finance}

We now deal with the problem of the yield of an American game, or recallable, option under Knightian uncertainty. First let us briefly describe that kind of option.

In a financial market assume we have a risky asset whose dynamic is given by

$$
d S_{t}=S_{t}\left(\mu d t+\nu d B_{t}\right), \quad t \leq T
$$

For the sake of simplicity we assume $\nu, \mu>0$ are real constants and $\left(B_{t}\right)_{t \leq T}$ is a 1-dimensional Brownian motion on the probability space $(\Omega, \mathscr{F}, P)$.

The usual American option is a contract between a trader and a broker who are, respectively, the buyer and the seller of the option. The trader pays a premium and obtains the right to ask for a wealth $\left(L_{t}\right)_{t \leq T}$ when he decides within a period of time $[0, T]$. The process $L$ is called the payoff of the option (e.g., $L_{t}=\left(S_{t}-K\right)^{+}$where $S$ is the dynamic of the asset which bears the option and $K$ the strike) and $T$ its maturity. So the main problems are the value of the option, the optimal time when the trader should exercise his option, the existence of a hedging strategy for the broker, and so forth. In the framework of those options, the broker has no right other than to provide the wealth for the trader when the latter decides to exercise his option.

An American game option is a standard American option where the broker is allowed to cancel the contract which binds him to the trader. In a way, he recalls the option. But in that case, he pays what the trader of the option would have earned if he had exercised his option at the same time plus a money penalty.

The motivations of those options is that insurance companies, which usually are the sellers of options, try to protect themselves against some events which could threaten their existence when facing charges which they cannot predict and bear.

The problem of pricing a recallable option in a complete financial market has been considered and solved in [8]. In this work we just focus on the yield of such an option under Knightian uncertainty.

So assume we have a trader $c_{1}$ who buys the recallable option and a broker $c_{2}$ who sells it. On the other hand, suppose that the option is on the risky asset whose dynamic is given by $\left(S_{t}\right)_{t \leq T}$. As said previously, if

(i) the trader decides first to exercise the option at a stopping time $\sigma$, he makes a profit which is equal to $e^{L_{\sigma}}$ (e.g., $L_{t}=\left(S_{t}-K\right)^{+}$where $K$ is the strike);

(ii) the broker decides first to cancel the option at $\tau$, he pays the trader an amount equal to $e^{U_{\tau}}$;

(iii) both decide to go up to the maturity $T$ of the option, $c_{1}$ earns $e^{\xi}$ paid by $c_{2}$.

The processes $L, U$ and the random variable $\xi$ are the same as in the previous section. On the other hand the difference $U-L$ is the money penalty that $c_{2}$ pays for his decision to cancel the option. Therefore the yield of the option when $c_{1}$ (resp., $c_{2}$ ) exercises (resp., cancels) at $\sigma$ (resp., $\tau$ ) is given by

$$
J(\tau, \sigma)=E\left[\exp \left\{L_{\sigma} 1_{[\sigma \leq \tau<T]}+U_{\tau} 1_{[\tau<\sigma]}+\xi 1_{[\tau=\sigma=T]}\right\}\right] .
$$


However rationality of the behaviors of the broker and the trader, implies that the yield of the option is given by

$$
V=\underset{\tau \geq 0}{\operatorname{essinf}} \underset{\sigma \geq 0}{\operatorname{esssup}} J(\tau, \sigma)=\underset{\sigma \geq 0}{\operatorname{esssup}} \underset{\tau \geq 0}{\operatorname{essinf}} J(\tau, \sigma)
$$

Knightian uncertainty assumes that we are not sure that the probability under which the market will evolve is $P$. It could be $P$ but it could also be another probability not far from $P$. Let $P^{\vartheta}$ be such a probability. The parameter $\vartheta=\left(\vartheta_{s}\right)_{s \leq T}$ is a $\mathscr{P}$-measurable stochastic process valued in a compact set $[-\kappa, \kappa] ; \kappa$ is called the degree of Knightian uncertainty and the set of those $\vartheta^{\prime} s$ is denoted by $\Theta$. Closeness of $P^{\vartheta}$ to $P$ leads us to assume that $P^{9}$ is absolutely continuous with respect to $P$ and its density function is given by

$$
\frac{d P^{\vartheta}}{d P}=\exp \left\{-\int_{0}^{T} \nu^{-1} \vartheta_{s} d B_{s}-\frac{1}{2}\left|\nu^{-1} \vartheta_{s}\right|^{2} d s\right\}
$$

Therefore the process $\left(S_{t}\right)_{t \leq T}$ of (5.1) satisfies the following: for any $t \leq T$,

$$
d S_{t}=\left(\mu-\vartheta_{t}\right) S_{t} d t+\nu S_{t} d B_{t}^{\vartheta}
$$

where $\left(B_{t}^{9}=B_{t}+\int_{0}^{t} v^{-1} \vartheta_{s}\right)_{t \leq T}$ is a Brownian motion under $P^{\vartheta}$. In a way this means that the yield of the risky asset in a short time interval $d t$ given $\mathscr{F}_{t}$ is equal to $\left(\mu-\vartheta_{t}\right) S_{t} d t$. For more detail on Knightian uncertainty one can refer to [18].

Now once again the behavior of the trader as well as that of the broker are rational, therefore the minimum and maximum yields of the option are given respectively by

$$
\begin{aligned}
Y^{\min } & =\inf _{\vartheta \in \Theta} \underset{\tau \geq 0}{\operatorname{essinf}} \underset{\sigma \geq 0}{\operatorname{esssup}} E^{\vartheta}\left[\exp \left\{L_{\sigma} 1_{[\sigma \leq \tau<T]}+U_{\tau} 1_{[\tau<\sigma]}+\xi 1_{[\tau=\sigma=T]}\right\}\right], \\
Y^{\max } & =\sup _{\vartheta \in \Theta} \operatorname{essinf} \underset{\tau \geq 0}{\operatorname{esssup}} E_{\sigma \geq 0}^{\vartheta}\left[\exp \left\{L_{\sigma} 1_{[\sigma \leq \tau<T]}+U_{\tau} 1_{[\tau<\sigma]}+\xi 1_{[\tau=\sigma=T]}\right\}\right] ;
\end{aligned}
$$

$E^{9}$ is the expectation under $P^{9}$.

Now we are going to characterize $Y^{\min }$ and $Y^{\max }$ via BSDEs which we have studied in Sections 2 and 3. Actually let $\theta \in[-\kappa, \kappa], z \in \mathbb{R}$, and $H(t, \theta, z)=-(\theta / \nu) z$. For $z \in \mathbb{R}$, let $\theta^{1}(z)=\kappa 1_{[z \leq 0]}-\kappa 1_{[z>0]}$ and $\theta^{2}(z)=-\theta^{1}(z)$. Therefore $\theta^{1}$ and $\theta^{2}$ verify

$$
H\left(t, \theta^{1}(z), z\right)=\sup _{\theta \in[-\kappa, \kappa]} H(t, \theta, z), \quad H\left(t, \theta^{2}(z), z\right)=\inf _{\theta \in[-\kappa, \kappa]} H(t, \theta, z) .
$$

Obviously the functions which with $z$ associate $H\left(t, \theta^{1}(z), z\right)$ and $H\left(t, \theta^{2}(z), z\right)$, respectively, are continuous with linear growth. Then we have the following result whose proof is a direct consequence of Theorem 4.1 . 

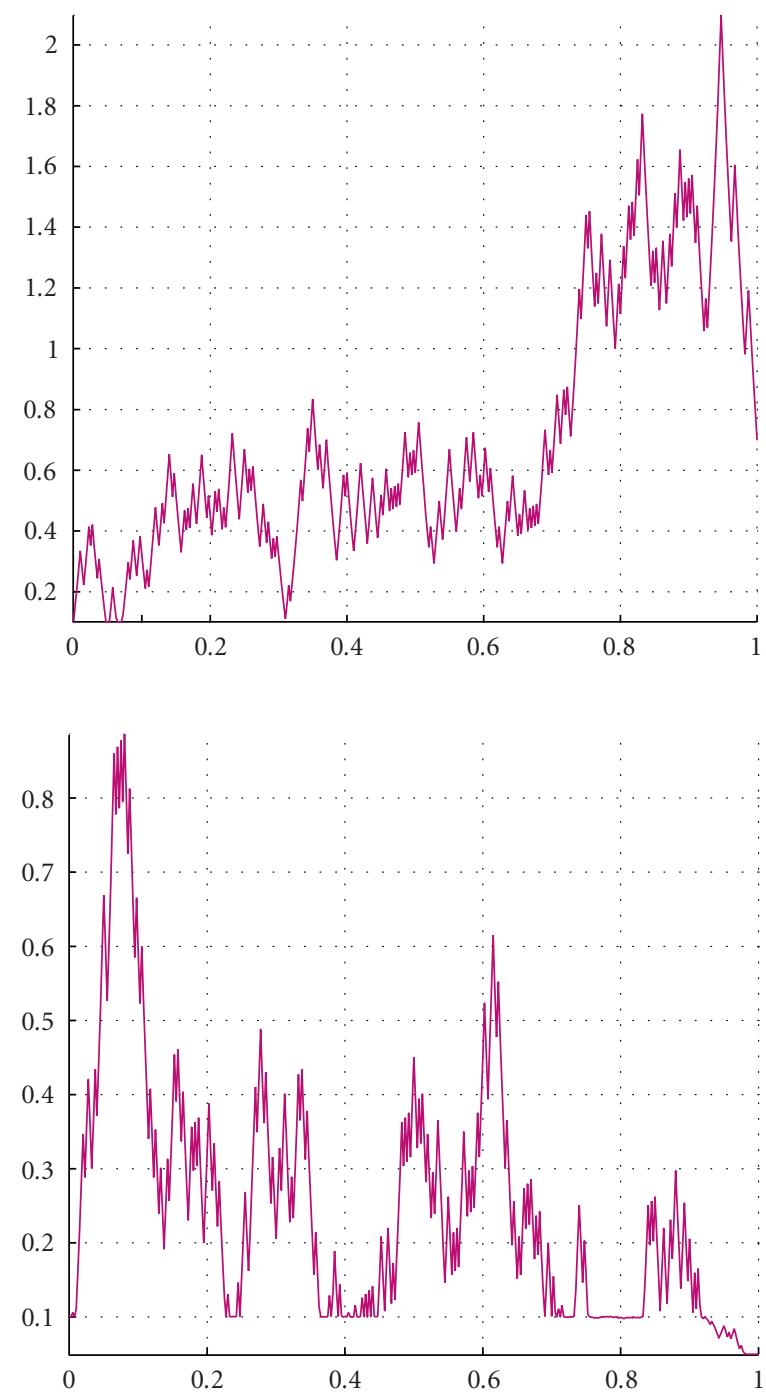

Figure 5.1. Simulation of paths of $\tilde{Y}$ and $\bar{Y}\left(\tilde{Y}_{0}=0\right.$ and $\left.\bar{Y}_{0}=0.1\right)$.

Theorem 5.1. Let $\left(\bar{Y}, \bar{Z}, \bar{K}^{+}, \bar{K}^{-}\right)$(resp., $\left.\left(\tilde{Y}, \widetilde{Z}, \widetilde{K}^{+}, \widetilde{K}^{-}\right)\right)$be the solution of the BSDE associated with $\left(H\left(t, \theta^{1}(z), z\right)+(1 / 2)|z|^{2}, \xi, L, U\right)$ (resp., $\left.\left(H\left(t, \theta^{2}(z), z\right)+(1 / 2)|z|^{2}, \xi, L, U\right)\right)$, then

$$
Y^{\max }=\exp \bar{Y}_{0}, \quad Y^{\min }=\exp \tilde{Y}_{0} .
$$

Figure 5.1 shows the simulations of paths of $\tilde{Y}$ and $\bar{Y}$. 


\section{Acknowledgment}

We thank M. Xu for the help she provided us when dealing with the simulations.

\section{References}

[1] K. Bahlali, S. Hamadène, and B. Mezerdi, Backward stochastic differential equations with two reflecting barriers and quadratic growth coefficient, Stochastic Processes and their Applications 115 (2005), no. 7, 1107-1129.

[2] V. E. Beneš, Existence of optimal strategies based on specified information, for a class of stochastic decision problems, SIAM Journal on Control and Optimization 8 (1970), 179-188.

[3] J. Cvitanić and I. Karatzas, Backward stochastic differential equations with reflection and Dynkin games, The Annals of Probability 24 (1996), no. 4, 2024-2056.

[4] N. El-Karoui and S. Hamadène, BSDEs and risk-sensitive control, zero-sum and nonzero-sum game problems of stochastic functional differential equations, Stochastic Processes and their Applications 107 (2003), no. 1, 145-169.

[5] N. El-Karoui, C. Kapoudjian, E. Pardoux, S. Peng, and M. C. Quenez, Reflected solutions of backward SDE's, and related obstacle problems for PDE's, The Annals of Probability 25 (1997), no. 2, 702-737.

[6] N. El-Karoui, E. Pardoux, and M. C. Quenez, Reflected backward SDEs and American options, Numerical Methods in Finance (L. Robers and D. Talay, eds.), Publ. Newton Inst., Cambridge University Press, Cambridge, 1997, pp. 215-231.

[7] N. El-Karoui, S. Peng, and M. C. Quenez, Backward stochastic differential equations in finance, Mathematical Finance. An International Journal of Mathematics, Statistics and Financial Economics 7 (1997), no. 1, 1-71.

[8] S. Hamadène, Mixed zero-sum differential game and American game options, preprint, Université du Maine, 2004.

[9] S. Hamadène and M. Hassani, BSDE's with two reflecting barriers: the general result, Probabability Theory and Related Fields 132 (2005), 237-264.

[10] S. Hamadène and J. P. Lepeltier, Backward equations, stochastic control and zero-sum stochastic differential games, Stochastics and Stochastics Reports 54 (1995), no. 3-4, 221-231.

[11] _ Zero-sum stochastic differential games and backward equations, Systems \& Control Letters 24 (1995), no. 4, 259-263.

[12] _ Reflected BSDEs and mixed game problem, Stochastic Processes and their Applications 85 (2000), no. 2, 177-188.

[13] S. Hamadène, J. P. Lepeltier, and A. Matoussi, Double barriers reflected backward SDE's with continuous coefficients, Pitman Research Notes in Mathematics Series 364 (1997), 115-128.

[14] S. Hamadène, J. P. Lepeltier, and S. Peng, BSDE with continuous coefficients and application to Markovian nonzero-sum stochastic differential games, Pitman Research Notes in Mathematics Series (N. El-Karoui and L. Mazliak, eds.), vol. 364, Longman, London, 1997, pp. 161-175.

[15] S. Hamadène, J. P. Lepeltier, and Z. Wu, Infinite horizon reflected backward stochastic differential equations and applications in mixed control and game problems, Probability and Mathematical Statistics 19 (1999), no. 2, 211-234.

[16] S. Hamadène and Y. Ouknine, Reflected backward stochastic differential equation with jumps and random obstacle, Electronic Journal of Probability 8 (2003), no. 2, 1-20.

[17] I. Karatzas and S. E. Shreve, Brownian Motion and Stochastic Calculus, Graduate Texts in Mathematics, vol. 113, Springer, New York, 1991.

[18] G. N. Kiohiko and O. Hiroyuki, Irreversible investment and Knightian uncertainty, CIRJE FSeries, CIRJE-F-176, CIRJE, Faculty of Economics, University of Tokyo, 2002.

[19] M. Kobylanski, Backward stochastic differential equations and partial differential equations with quadratic growth, The Annals of Probability 28 (2000), no. 2, 558-602. 
[20] M. Kobylanski, J. P. Lepeltier, M. C. Quenez, and S. Torres, Reflected BSDE with superlinear quadratic coefficient, Probability and Mathematical Statistics 22 (2002), no. 1, 51-83.

[21] A. N. Kolmogorov and S. V. Fomin, Introductory Real Analysis, Dover Publications, New York, 1975.

[22] J. P. Lepeltier and J. San Martin, Backward stochastic differential equations with continuous coeffcient, Statistics \& Probability Letters 32 (1997), no. 4, 425-430.

[23] J. P. Lepeltier and J. San Martin, Existence for BSDE with superlinear-quadratic coefficient, Stochastics and Stochastics Reports 63 (1998), no. 3-4, 227-240.

[24] J. Mémin, S. Peng, and M. Xu, Convergence of solutions of discrete reflected backward SDE's and simulations, preprint of Institut de Recherche Mathématique de Rennes, Rennes, 2003.

[25] E. Pardoux, BSDEs, weak convergence and homogenization of semilinear PDEs, Nonlinear Analysis, Differential Equations and Control (Montreal, QC, 1998) (F. Clarke and R. Stern, eds.), NATO Sci. Ser. C Math. Phys. Sci., vol. 528, Kluwer Academic, Dordrecht, 1999, pp. 503-549.

[26] E. Pardoux and S. Peng, Adapted solution of a backward stochastic differential equation, Systems \& Control Letters 14 (1990), no. 1, 55-61.

[27] _ Backward stochastic differential equations and quasilinear parabolic partial differential equations, Stochastic Partial Differential Equations and Their Applications (Charlotte, NC, 1991) (B. Rozovskii and R. Sowers, eds.), Lecture Notes in Control and Inform. Sci., vol. 176, Springer, Berlin, 1992, pp. 200-217.

[28] __ Backward doubly stochastic differential equations and systems of quasilinear SPDEs, Probability Theory and Related Fields 98 (1994), no. 2, 209-227.

[29] S. Peng, Probabilistic interpretation for systems of quasilinear parabolic partial differential equations, Stochastics and Stochastics Reports 37 (1991), no. 1-2, 61-74.

[30] D. Revuz and M. Yor, Continuous Martingales and Brownian Motion, Grundlehren der Mathematischen Wissenschaften, vol. 293, Springer, Berlin, 1991.

[31] R. Rouge and N. El-Karoui, Pricing via utility maximization and entropy, Mathematical Finance. An International Journal of Mathematics, Statistics and Financial Economics 10 (2000), no. 2, 259-276.

S. Hamadène: Laboratoire de Statistique et Processus, Université du Maine, 72085 Le Mans Cedex 9, France

E-mail address: hamadene@univ-lemans.fr

I. Hdhiri: Laboratoire de Statistique et Processus, Université du Maine,

72085 Le Mans Cedex 9, France

E-mail address: ibtissam.hdhiri@univ-lemans.fr 


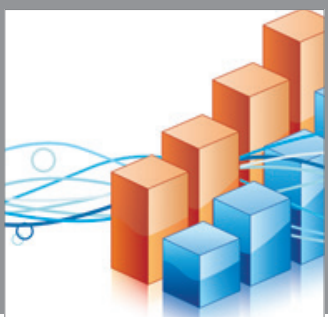

Advances in

Operations Research

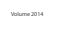

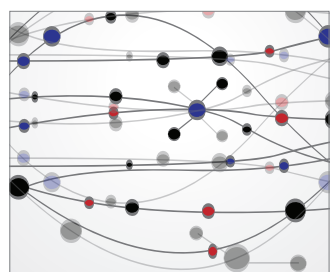

\section{The Scientific} World Journal
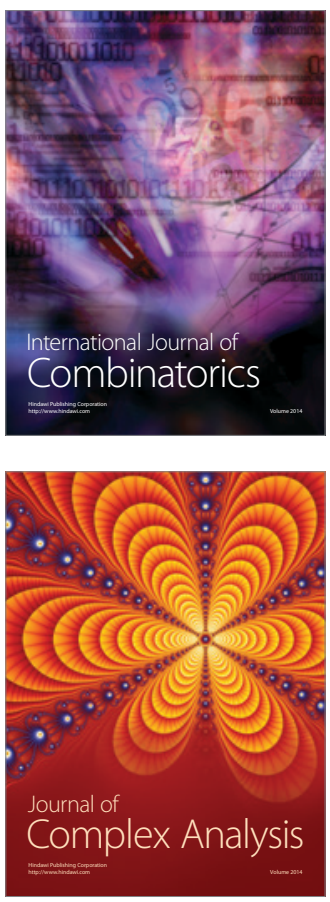

International Journal of

Mathematics and

Mathematical

Sciences
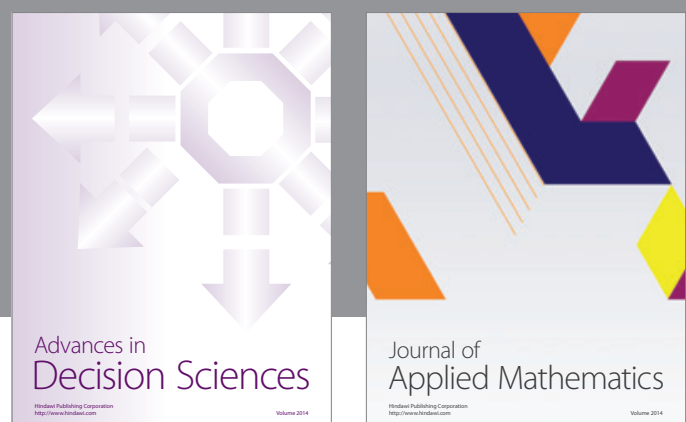

Journal of

Applied Mathematics
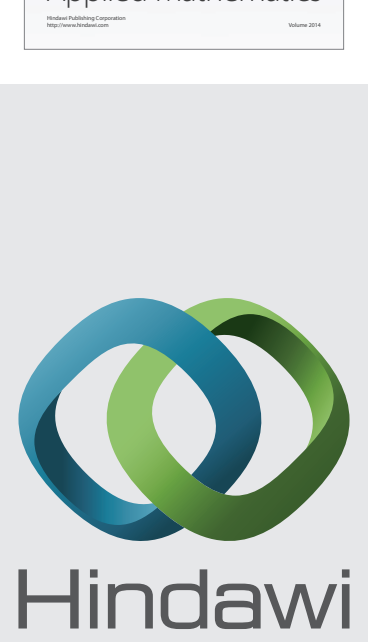

Submit your manuscripts at http://www.hindawi.com
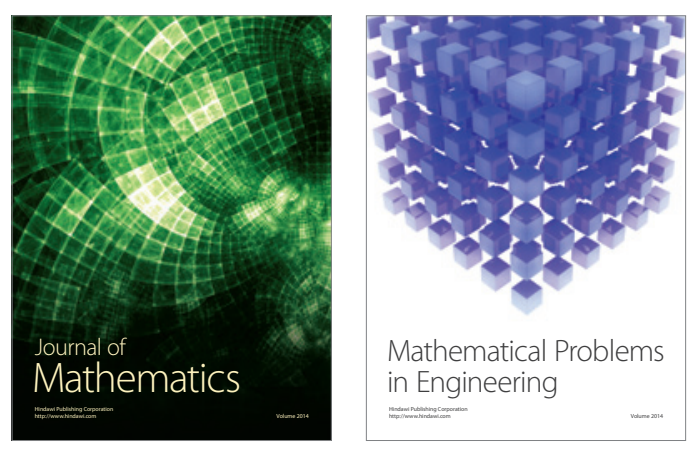

Mathematical Problems in Engineering
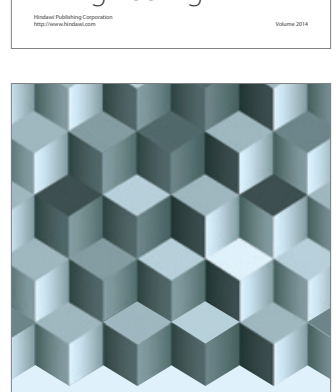

Journal of

Function Spaces
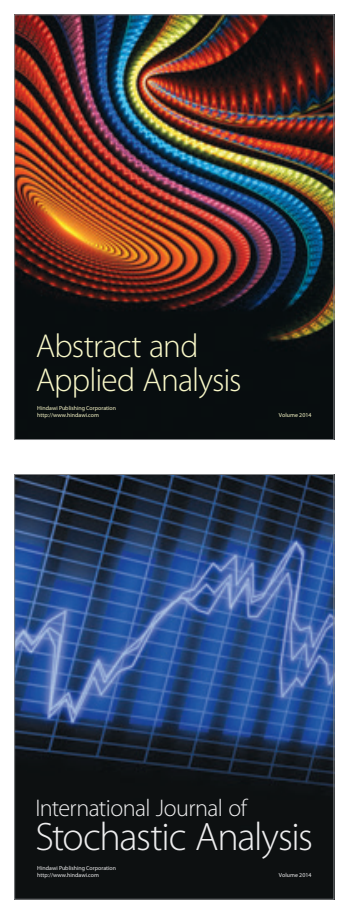

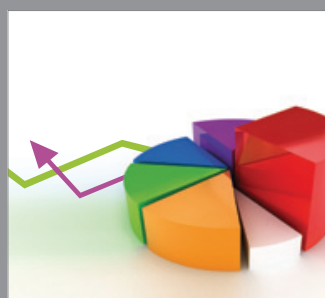

ournal of

Probability and Statistics

Promensencen
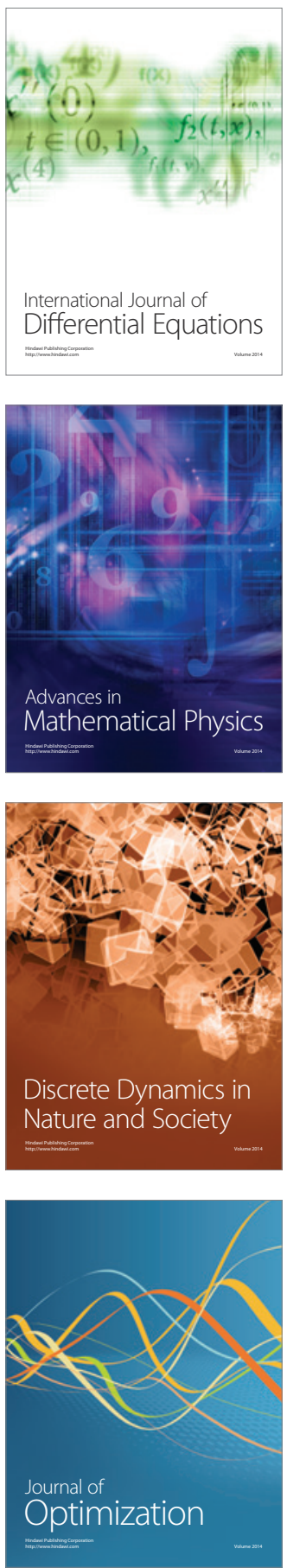